\title{
Equity Capital as a Safety Cushion in the US Banking Sector
}

\author{
Raymond A. K. Cox ${ }^{1}$, Randall K. Kimmel ${ }^{1} \&$ Grace W. Y. Wang ${ }^{2}$ \\ ${ }^{1}$ Department of Accounting and Finance, School of Business and Economics, Thompson Rivers University, \\ Kamloops, Canada \\ ${ }^{2}$ Department of Maritime Administration, Texas A\&M University, Galveston, USA \\ Correspondence: Raymond A. K. Cox, School of Business and Economics, 900 McGill Road, Kamloops, British \\ Columbia, V2C 0C8, Canada. Tel: 01-250-852-6387. E-mail: rcox@tru.ca
}

Received: July 9, 2016

Accepted: July 29, 2016

Online Published: August 25, 2016

doi:10.5539/ijef.v8n9p50

URL: http://dx.doi.org/10.5539/ijef.v8n9p50

\begin{abstract}
The incidence of US bank failures soared in the financial crisis and economic recession starting in 2008 . Financial regulations promulgated by the Federal Reserve and issued through the Basel III Accord raised the minimum equity capital requirements of banks. The intent of the increase in equity capital was to serve as a greater safety cushion to reduce the probability of failure. The purpose of this study is to examine the financial statement variables that distinguish failed (zero equity capital) and nonfailed US banks. The methods employed to investigate our research question are: 1 . univariate t-test, and 2. tobit regression analysis with equity capital as the dependent variable. Our results show that the factors explaining equity capital include real estate loans to assets, equity capital to total assets, log of total assets, return on equity, loan loss allowance to total loans, non-performing loans to total assets, total loans to total assets, mortgage-backed securities to total assets, total short-term debt securities to total assets, net gains on sales of loans to total non-interest income, and insured deposits to total deposits. Bank management and financial regulators need to focus on these financial characteristics to ensure adequate equity capital as a safety cushion.
\end{abstract}

Keywords: problem banks, financial crisis, tobit analysis, equity capital, Basel III

\section{Introduction}

During the financial crisis and economic recession of 2008 to 2010 financial institution failures soared in the US especially in the banking sector. Investors, analysts and regulators scrutinized bank's financial statements in search of the underlying factors leading to bankruptcy. The financial characteristics examined included the asset mix (lending), earnings profile (interest and fees income, expense composition), liquidity, market risk susceptibility, and the capacity of equity capital to act as a safety cushion absorbing the operating loss shocks.

Governments and financial regulators are compelled to respond to the rise in bank failures and downturn in the economy. Actions taken to combat this financial storm, by the Federal Reserve, included lowering short-term interest rates, increasing loans to banks, expanding the list of collateral eligible to secure loans, and bailing out related financial institutions such as AIG who insured much of the credit default swap market. The federal government responded by reducing corporate income tax rates, adding refunds to individuals, increasing spending and changing legislation to make house foreclosures more difficult resulting in a greater likelihood of refinancing.

Further, financial regulation occurred at the international level, in particular, the Basel III Accord with respect to equity capital on the bank balance sheet. The minimum common equity tier 1 (CET1) to risk-weighted assets (RWA) ratio is 6 percent and 7 percent as of 2015 and 2019 respectively. A supplementary equity capital amount of as much as 2.5 percent can be required during periods of high growth. In conjunction with the international equity capital standards the Federal Reserve mandated a minimum financial leverage ratio (Tier 1 Capital to Total Assets) for US banks of 5 percent for holding companies and 8 percent for systemically important financial institutions (SIFI). In 2016 the eight US SIFIs are Bank of America, Bank of New York Mellon, Citigroup, Goldman Sachs, JP Morgan Chase, Morgan Stanley, State Street and Wells Fargo.

When banks have suffered losses reducing their equity capital to the point of having an inadequate safety cushion their regulator closes them. As outlined by Walter (2004) the closure decision is made by the Office of the Comptroller of the Currency for national-chartered banks, State Government Agencies for state-chartered 
banks, and by the Office of Thrift Supervision (dissolved in 2011) for savings associations having a federal government charter. The Federal Deposit Insurance Corporation (FDIC) can decide to close a state-chartered bank without the approval of the State Government Agency. The FDIC typically is appointed the receiver for the closed bank and can choose to conduct a deposit payoff or purchase and assumption.

In this study, first, we investigate the financial statement variables that distinguish failed and nonfailed US banks using a univariate t-test. Second, tobit regression analysis is shown to explain the financial characteristics associated with the amount of equity capital during the financial crisis of 2008 to 2010 . Third, some suggestions are made for management on how to operate the bank to augment its equity capital and thereby strengthen its safety cushion to face economic and financial market downturns.

The paper is comprised as follows. Section 2 reviews the literature. Section 3 outlines the data, sample, and hypothesis. Section 4 presents the methodology. Section 5 details and discusses the empirical results. Finally, section 6 concludes the study.

\section{Literature Review}

Sinkey $(1974,1975)$ researched problem and non-problem banks finding that the growth in equity capital was not commensurate with the asset growth rate. Hutchison and Cox (2007) demonstrated a positive relation between financial leverage and the return on equity and return on assets. Brunnermeier and Pedersen (2009) as well as Shleifer and Vishny (2010) found that in economic downturns high financial leverage banks must liquidate their loans at a loss reducing their equity capital to the point of bank failure. James (1991) found the losses associated with the sale of closed bank assets to be 40 percent of book value. Acharya et al. (2010) showed that restricted debt capacity, partially caused by low equity capital, further increased the probability of bank failure. Wagner (2007) discovered banks that sell their loans also have a higher risk asset portfolio leading to instability. Moreover, Uzun and Web (2007) presented results that banks who securitize assets are larger and inversely related to the degree of equity capital.

Early warning systems of problem banks have been studied by Gonzalez-Hermosillo (1999), Cihak and Schaeck (2010), and Cole and White (2012), using the CAMELS approach, finding inadequate equity capital was a predictor of failure. CAMELS is the acronym for capital adequacy, asset quality, management quality, earnings, liquidity and sensitivity to the market. Cox and Wang (2014), utilizing discriminant analysis, discovered low equity capital as a factor in US bank failures in the 2008 to 2010 financial crisis. Mare (2015) discovered the contribution of macroeconomic factors to the forecasting of small Italian bank failures, leading to the notion that capital requirements should consider the stage of the business cycle in a countercyclical fashion. Ho et al. (2016) presented evidence that overconfident chief executive officers were more likely to increase the debt ratio prior to a crisis culminating in higher failure rates.

\section{Data, Sample, and Hypothesis}

Financial statement data for the variables in the models come from the Federal Deposit Insurance Corporation. House price index information (hpindexsa) comes from the Federal Housing Finance Agency and percentage change in personal income (pigrow) comes from the Bureau of Economic Analysis. We access the Bank Data and Statistics under Industry Analysis data assembled by the FDIC from the call reports of US banks for the 2005 to 2010 period. We gather information to calculate 29 independent variables.

The explanatory variables and the predicted relation between them and the dependent variable of bank equity capital is provided in Table 1. We examine five models explaining bank equity capital. The five models delineate different financial characteristic combinations explaining equity capital. The rationale for the different models revolve around the asset mix (loan type), growth of loans and quality of loans.

Book common equity is used as a proxy for market equity. When the common equity of a bank decreases to such an extent that it is negative or zero the bank is closed. There are other banks with very low equity capital that are closed by the respective regulator. In these cases the equity value is worthless. All surviving banks continue to have a positive equity capital balance. 
Table 1. Variables and descriptions

\begin{tabular}{|c|c|c|c|}
\hline Variable & Description & Expected Sign & Rationale \\
\hline ciloan & $\begin{array}{l}\text { commercial and industrial loans to total } \\
\text { assets }\end{array}$ & negative & Like comm_real. \\
\hline mul_family & $\begin{array}{l}\text { multifamily residential real estate loans } \\
\text { to real estate loans }\end{array}$ & positive & $\begin{array}{l}\text { People continue to have a need for housing in meltdowns and } \\
\text { recession. }\end{array}$ \\
\hline sig_family & $\begin{array}{l}1-4 \text { family residential loans to real estate } \\
\text { loans }\end{array}$ & positive & Similar to mul_family. \\
\hline trade_ast & trading account assets to total assets & uncorrelated & Assets owned by customers. \\
\hline brokdep & brokered deposits to total deposits & negative & $\begin{array}{l}\text { This is hot money from brokers raising deposits from high } \\
\text { interest certificates of deposit indicative of a high risk bank. }\end{array}$ \\
\hline chargeoff & net charge offs to average loans & negative & This is the recognised bad debt experience. \\
\hline comm_real & $\begin{array}{l}\text { commercial real estate loans to real } \\
\text { estate loans }\end{array}$ & negative & $\begin{array}{l}\text { These assets are income-producing properties focusing on } \\
\text { financing commercial real estate developers. They are } \\
\text { sensitive to economic downturns. }\end{array}$ \\
\hline cons_devlp & $\begin{array}{l}\text { construction and land development } \\
\text { loans to real estate loans }\end{array}$ & negative & These are risky assets sensitive to the business cycle. \\
\hline foreclosure & $\begin{array}{l}\text { real estate acquired of other real estate } \\
\text { owned to total assets }\end{array}$ & negative & $\begin{array}{l}\text { This is the process to repossess the security (houses) pledged } \\
\text { for loans. }\end{array}$ \\
\hline loanast & Total loans to total assets & positive & $\begin{array}{l}\text { The higher the level of loans and lease financing receivables } \\
\text { to total assets, the safer the bank's portfolio. }\end{array}$ \\
\hline loansale & $\begin{array}{l}\text { net gains on sales of loans to total } \\
\text { non-interest income }\end{array}$ & negative & $\begin{array}{l}\text { Banks that are selling their loans are in need of liquidity which } \\
\text { is connected with poor operating performance. }\end{array}$ \\
\hline lossallow & loan loss allowance to total loans & negative & Reflects expected bad debt expense. \\
\hline pastdue & non-performing loans to total assets & negative & Similar to chargeoff \\
\hline capital & equity capital to total assets & positive & $\begin{array}{l}\text { The higher this ratio the greater financial strength and ability } \\
\text { to weather the storm in dire times. }\end{array}$ \\
\hline cash & $\begin{array}{l}\text { cash and due from depository } \\
\text { institutions to total assets }\end{array}$ & positive & If this ratio is too low it implies illiquidity. \\
\hline debt_sec & $\begin{array}{l}\text { total short-term debt securities to total } \\
\text { assets }\end{array}$ & positive & These include government securities owned. \\
\hline deploan & $\begin{array}{l}\text { loans to depository institutions to total } \\
\text { assets }\end{array}$ & positive & These are assets to high-quality institutions. \\
\hline idloan & loans to individuals to total assets & positive & $\begin{array}{l}\text { These loans include credit cards whose risk can be } \\
\text { micromanaged with the credit limits and short maturity } \\
\text { coupled with high income from interest and fees. }\end{array}$ \\
\hline insureddep & Insured deposits to total deposits & positive & $\begin{array}{l}\text { The greater the percentage of insured deposits the lower the } \\
\text { number of high-value deposits being monitored by their } \\
\text { owners leading to lower market discipline. }\end{array}$ \\
\hline interbank & interbank deposits to total deposits & positive & $\begin{array}{l}\text { Presumably banks monitor the default risk of the banks they } \\
\text { deposit in. Thus, a high inter bank is associated with } \\
\text { confidence of other banks in the risk of the deposit bank. }\end{array}$ \\
\hline loar & f total loans and leases & posit & $\begin{array}{l}\text { High loan growth rates typically indicate higher credit risk. } \\
\text { However, once the economy has entered into a crisis weaker } \\
\text { banks susceptible to failure will abandon loan growth. }\end{array}$ \\
\hline MBS & $\begin{array}{l}\text { mortgage-backed securities to total } \\
\text { assets }\end{array}$ & positive & $\begin{array}{l}\text { As stated in the literature, before this crisis MBS were viewed } \\
\text { as gilt-edge assets. On the other hand, MBS is of long duration } \\
\text { exposing the holder to interest rate risk and heavy losses if } \\
\text { rates increase. However, typically in financial crisis regulators } \\
\text { combat the calamity by injecting liquidity and decreasing } \\
\text { interest rates. }\end{array}$ \\
\hline non_income & non-interest income to total income & positive & $\begin{array}{l}\text { This variable generates a more stable income stream from } \\
\text { sources other than securities and loans. }\end{array}$ \\
\hline off-bal & $\begin{array}{l}\text { off-balance sheet derivatives to total } \\
\text { assets }\end{array}$ & positive & Normally sophisticated banks engage in derivatives. \\
\hline
\end{tabular}




\begin{tabular}{|c|c|c|c|}
\hline realloan & real estate loans to total assets & positive & $\begin{array}{l}\text { Prior to the housing asset bubble bursting in the time period of } \\
\text { this study loans secured by real estate were considered to be } \\
\text { safe, secured by a mortgage on a consumer's primary } \\
\text { residence. }\end{array}$ \\
\hline roa & return on assets & positive & High roa means high profitability. \\
\hline sec_asset & securities to total assets & positive & Highly liquid assets \\
\hline size & $\log$ of total assets & positive & $\begin{array}{l}\text { In the past most failures were small banks. That and some } \\
\text { banks are too big to fail. }\end{array}$ \\
\hline tier1 & $\begin{array}{l}\text { Tier } 1 \text { risk-based capital to total } \\
\text { risk-weighted assets }\end{array}$ & positive & Along the same lines as capital. \\
\hline hpindexsa & Home price index seasonal adjusted & & $\begin{array}{l}\text { Quarterly All-Transactions Home Price Indexes (Estimated } \\
\text { using Sales Prices and Appraisal Data) that estimates the } \\
\text { percentage change in home values. Source: the Federal } \\
\text { Housing Finance Agency. }\end{array}$ \\
\hline pigrow & Growth of personal income & & $\begin{array}{l}\text { Percent change of the personal income. Source: Bureau of } \\
\text { Economic Analysis. }\end{array}$ \\
\hline
\end{tabular}

\section{Methodology}

The first methodology is comparing banks that had a positive amount of capital (common equity $>0$ ) to the banks that had zero equity capital. A univariate $t$-test for mean differences for each of the 29 independent variables listed in Table 1 is conducted.

The second methodology is the use of tobit regression analysis. Tobit regression was created by Tobin (1958). The suitability of tobit rests with the empirics of having a dependent variable with a limiting value typically zero. The limited value is the censored bound versus the upside of having an unlimited value called the uncensored value. In Tobit failed banks that are closed are censored. The efficacy of tobit, as opposed to ordinary least squares (OLS), regression, has been examined by McDonald and Moffitt (1980), Foster and Kalenkoski (2013), and Stewart (2013) among others.

There are five tobit regression equations representing five hypothesized models to explain the financial characteristics of banks with an equity capital amount.

Model 1:

$$
\text { Equity }=\beta_{0}+\beta_{1} \text { capital }+\beta_{2} \text { deploan }+\beta_{3} \text { idloan }+\beta_{4} \text { loangrowth }+\beta_{5} \text { reallaon }+\beta_{6} \text { roa }+\beta_{7} \text { size }
$$

Model 2:

$$
\begin{gathered}
\text { Equity }=\beta_{0}+\beta_{1} \text { capital }+\beta_{2} \text { ciloan }+\beta_{3} \text { mulfamily }+\beta_{4} \text { sigfamily }+\beta_{5} \text { commreal }+\beta_{6} \text { consdevlp } \\
+\beta_{7} \text { roa }+\beta_{8} \text { size }
\end{gathered}
$$

Model 3:

$$
\begin{gathered}
\text { Equity }=\beta_{0}+\beta_{1} \text { capital }+\beta_{2} \text { chargeoff }+\beta_{3} \text { foreclosure }+\beta_{4} \text { lossallow }+\beta_{5} \text { pastdue }+\beta_{6} \text { loangrowth } \\
+\beta_{7} \text { realloan }+\beta_{8} \text { roa }+\beta_{9} \text { size }
\end{gathered}
$$

Model 4:

$$
\begin{gathered}
\text { Equity }=\beta_{0}+\beta_{1} \text { capital }+\beta_{2} \text { loanast }+\beta_{3} \text { loansale }+\beta_{4} \text { lossallow }+\beta_{5} \text { pastdue }+\beta_{6} \text { debtsec } \\
+\beta_{7} \text { insureddep }+\beta_{8} M B S+\beta_{9} \text { realloan }+\beta_{10} \text { roa }+\beta_{11} \text { size }
\end{gathered}
$$

Model 5:

$$
\begin{aligned}
\text { Equity }= & \beta_{0}+\beta_{1} \text { capital }+\beta_{2} \text { loanast }+\beta_{3} \text { lossallow }+\beta_{4} \text { pastdue }+\beta_{5} \text { debtsec }+\beta_{6} M B S \\
& +\beta_{7} \text { realloan }+\beta_{8} \text { roa }+\beta_{9} \text { size }+\beta_{10} \text { hpindexs }+\beta_{11} \text { pigrow }
\end{aligned}
$$

The tobit regressions are run with rolling windows, consisting of four combinations of time (the first quarter of 2005, 2006, 2007, and 2008) for each of the four fixed window dependent variable forecasts $(2007,2008,2009$, and 2010).

\section{Results}

The results for the univariate t-tests are reported in Table 2 for 2007 Quarter 4 and Table 3 for 2008 Quarter 4. Clearly surviving banks have a significantly higher quantity of capital and tier 1 equity than banks that failed. The 
highly significant (alpha $\leq 0.01$ for each of the 2 years) variables with failed banks having a higher value than surviving banks are realloan, cons_devlp, mul_family, chargeoff, lossallow, pastdue, foreclose, size, brokdep, interbank, and loan_ast. The highly significant variables with a lower value for failed banks versus surviving banks are sig_family, idloan, loangrowth, capital, tier 1, roa, sec_asset, debt_sec, non_income, and cash. This is in line with our a priori expectations with the exception of mul_family, loanast, interbank, realloan and size. Following previous research we believed that high exposure to residential real estate loans, higher percentage of assets in loans, higher percentage of interbank loans, and larger banks in terms of total assets would be associated with higher equity levels and increased odds of survival, but during the crisis which began in 2008 these associations were reversed.

Table 2. Descriptive statistics and univariate t-test for mean differences (2007Q4)

\begin{tabular}{|c|c|c|c|c|c|c|c|c|c|}
\hline variable & $\begin{array}{l}\text { failed } \\
\text { banks }\end{array}$ & $\begin{array}{c}\text { Surviving } \\
\text { banks }\end{array}$ & $\begin{array}{c}\text { Difference } \\
\text { (t-stat) }\end{array}$ & & variable & $\begin{array}{l}\text { Failed } \\
\text { banks }\end{array}$ & $\begin{array}{c}\text { Surviving } \\
\text { banks }\end{array}$ & $\begin{array}{c}\text { Difference } \\
\text { (t-stat) }\end{array}$ & \\
\hline \multirow[t]{2}{*}{ realloan } & 64.18 & 47.38 & -16.8 & & size & 12.88 & 11.91 & -0.96 & \\
\hline & $(-14.60)$ & $(-19.86)$ & $(-13.35)$ & $* * *$ & & $(-1.61)$ & $(-1.38)$ & $(-7.58)$ & $* * *$ \\
\hline \multirow[t]{2}{*}{ cons_devlp } & 38.67 & 15.16 & -23.51 & & roa & -1.84 & 0.51 & 2.35 & \\
\hline & $(-22.16)$ & $(-15.41)$ & $(-12.45)$ & $* * *$ & & $(-5.39)$ & $(-5.97)$ & $(-5.49)$ & $* * *$ \\
\hline \multirow[t]{2}{*}{ comm_real } & 28.53 & 30.58 & 2.05 & & sec_asset & 12.78 & 20.07 & 7.29 & \\
\hline & $(-16.47)$ & $(-18.27)$ & $(-1.57)$ & & & $(-10.52)$ & $(-15.13)$ & $(-8.66)$ & $* * *$ \\
\hline \multirow[t]{2}{*}{ mul_family } & 4.97 & 2.82 & -2.15 & & trade_ast & 0.09 & 0.1 & 0.013 & \\
\hline & $(-7.97)$ & $(-5.88)$ & $(-3.42)$ & $* * *$ & & $(-0.39)$ & $(-1.43)$ & $(-0.39)$ & \\
\hline \multirow[t]{2}{*}{ sig_family } & 24.34 & 40.82 & 16.48 & & MBS & 5.14 & 6.32 & 1.18 & \\
\hline & $(-22.94)$ & $(-23.76)$ & $(-9.07)$ & $* * *$ & & $(-6.15)$ & $(-9.27)$ & $(-2.40)$ & $* *$ \\
\hline \multirow[t]{2}{*}{ Ciloan } & 8.84 & 9.35 & 0.51 & & off_bal & 1.97 & 3.46 & 1.49 & \\
\hline & $(-7.53)$ & $(-7.65)$ & $(-0.86)$ & & & $(-7.93)$ & $(-94.47)$ & $(-1.15)$ & \\
\hline \multirow[t]{2}{*}{ Idloan } & 1.64 & 4.67 & 3.03 & & debt_sec & 12.2 & 19.71 & 7.51 & \\
\hline & $(-2.07)$ & $(-6.74)$ & $(-16.96)$ & $* * *$ & & $(-10.36)$ & $(-14.93)$ & $(-9.07)$ & $* * *$ \\
\hline \multirow[t]{2}{*}{ deploan } & 0.012 & 0.071 & 0.06 & & loansale & 1.75 & 0.16 & -1.58 & \\
\hline & $(-0.11)$ & $(-1.19)$ & $(-3.54)$ & $* * *$ & & $(-5.11)$ & $(-61.11)$ & $(-1.99)$ & $* *$ \\
\hline \multirow[t]{2}{*}{ loangrowth } & 3.75 & 9.33 & 5.58 & & brokdep & 16.14 & 4.06 & -12.08 & \\
\hline & $(-15.94)$ & $(-165.30)$ & $(-2.49)$ & $* *$ & & $(-19.86)$ & $(-10.15)$ & $(-7.75)$ & $* * *$ \\
\hline \multirow[t]{2}{*}{ lossallow } & 1.85 & 1.29 & -0.56 & & interbank & 5.51 & 1.54 & -3.97 & \\
\hline & $(-1.33)$ & $(-1.48)$ & $(-5.28)$ & $* * *$ & & $(-13.02)$ & $(-7.74)$ & $(-3.88)$ & $* * *$ \\
\hline \multirow[t]{2}{*}{ chargeoff } & 0.36 & 0.11 & -0.25 & & non_income & 4.23 & 10.05 & 5.82 & \\
\hline & $(-0.72)$ & $(-0.40)$ & $(-4.38)$ & $* * *$ & & $(-22.03)$ & $(-17.51)$ & $(-3.35)$ & $* * *$ \\
\hline \multirow[t]{2}{*}{ pastdue } & 5.72 & 1.72 & -4 & & cash & 2.37 & 4.65 & 2.27 & \\
\hline & $(-5.57)$ & $(-1.80)$ & $(-9.15)$ & $* * *$ & & $(-2.06)$ & $(-5.55)$ & $(-13.18)$ & $* * *$ \\
\hline \multirow[t]{2}{*}{ foreclose } & 0.75 & 0.18 & -0.56 & & loan_ast & 76.24 & 66.2 & -10.04 & \\
\hline & $(-1.19)$ & $(-0.51)$ & $(-6.04)$ & $* * *$ & & $(-12.37)$ & $(-17.58)$ & $(-10.16)$ & $* * *$ \\
\hline \multirow[t]{2}{*}{ Capital } & 9.75 & 12.74 & 2.99 & & insureddep & 71.91 & 75.17 & 3.26 & \\
\hline & $(-4.35)$ & $(-9.68)$ & $(-8.35)$ & $* * *$ & & $(-16.19)$ & $(-16.06)$ & $(-2.55)$ & $* *$ \\
\hline \multirow[t]{2}{*}{ tier1 } & 10.85 & 22.1 & 11.25 & & & & & & \\
\hline & $(-4.21)$ & $(-116.18)$ & $(-8.41)$ & $* * *$ & & & & & \\
\hline
\end{tabular}

Note. We obtained the results by using the cross-sectional data of 2007Q4. Failure dummy variable defined as banks that failed in 2008-2009. We reported the mean of explanatory variables for surviving and failed banks in the first two columns. The standard deviations are in the parenthesis. We also present the difference in mean and the t-statistic in the third column which tests the mean difference of both sample banks. $*, * *$ and $* * *$ significant at the $10 \%, 5 \%$ and $1 \%$ level, variables are described in Table 1. 
Table 3. Descriptive statistics and univariate t-test for mean differences (2008Q4)

\begin{tabular}{|c|c|c|c|c|c|c|c|}
\hline variable & Failed banks & $\begin{array}{c}\text { Surviving } \\
\text { banks }\end{array}$ & $\begin{array}{r}\text { Difference } \\
\text { (t-stat) }\end{array}$ & variable & Failed banks & $\begin{array}{c}\text { Surviving } \\
\text { banks }\end{array}$ & $\begin{array}{r}\text { Difference } \\
\text { (t-stat) }\end{array}$ \\
\hline realloan & $\begin{array}{c}63.57 \\
(-13.78)\end{array}$ & $\begin{array}{c}48.45 \\
(-19.59)\end{array}$ & $\begin{array}{l}-15.13 \\
(18.06) * * *\end{array}$ & size & $\begin{array}{c}12.65 \\
(-1.36)\end{array}$ & $\begin{array}{c}11.98 \\
(-1.37)\end{array}$ & $\begin{array}{l}-0.67 * * * \\
(-8.30)\end{array}$ \\
\hline cons_devlp & $\begin{array}{c}31.95 \\
(-17.82)\end{array}$ & $\begin{array}{c}13.04 \\
(-12.59)\end{array}$ & $\begin{array}{l}-18.91 \\
(17.91) * * *\end{array}$ & roa & $\begin{array}{l}-7.65 \\
(-8.81)\end{array}$ & $\begin{array}{c}-0.22 \\
(-5.13)\end{array}$ & $\begin{array}{r}7.43 \text { *** } \\
(-14.31)\end{array}$ \\
\hline comm_real & $\begin{array}{c}33.13 \\
(-16.04)\end{array}$ & $\begin{array}{c}31.82 \\
(-18.47)\end{array}$ & $\begin{array}{l}-1.31 \\
(1.36)\end{array}$ & sec_asset & $\begin{array}{c}10.77 \\
(-8.65)\end{array}$ & $\begin{array}{c}19.8 \\
(-15.13)\end{array}$ & $\begin{array}{l}9.03 \text { *** } \\
(-16.89)\end{array}$ \\
\hline mul_family & $\begin{array}{c}5.27 \\
(-7.76)\end{array}$ & $\begin{array}{c}3.02 \\
(-5.89)\end{array}$ & $\begin{array}{l}-2.24 \\
(4.87) * * *\end{array}$ & trade_ast & $\begin{array}{c}0.003 \\
(-0.02)\end{array}$ & $\begin{array}{c}0.08 \\
(-1.35)\end{array}$ & $\begin{array}{l}0.08 \text { *** } \\
(-5.14)\end{array}$ \\
\hline sig_family & $\begin{array}{c}27.04 \\
(-20.62)\end{array}$ & $\begin{array}{c}41.19 \\
(-23.07)\end{array}$ & $\begin{array}{c}14.15 \\
(-11.42) * * *\end{array}$ & MBS & $\begin{array}{c}5.9 \\
(-6.65)\end{array}$ & $\begin{array}{c}7.85 \\
(-10.42)\end{array}$ & $\begin{array}{l}1.95 \text { *** } \\
(-4.79)\end{array}$ \\
\hline Ciloan & $\begin{array}{c}9.08 \\
(-7.42)\end{array}$ & $\begin{array}{c}9.2 \\
(-7.58)\end{array}$ & $\begin{array}{r}0.13 \\
(-0.29)\end{array}$ & off_bal & $\begin{array}{c}1.06 \\
(-4.36)\end{array}$ & $\begin{array}{c}6.74 \\
(-309.95)\end{array}$ & $\begin{array}{r}5.68 \\
(-1.54)\end{array}$ \\
\hline Idloan & $\begin{array}{c}1.66 \\
(-2.22)\end{array}$ & $\begin{array}{c}4.39 \\
(-6.60)\end{array}$ & $\begin{array}{c}2.73 \\
(-18.19) * * *\end{array}$ & debt_sec & $\begin{array}{c}10.6 \\
(-8.47)\end{array}$ & $\begin{array}{c}19.55 \\
(-14.96)\end{array}$ & $\begin{array}{c}8.94 \\
(-17.06)\end{array}$ \\
\hline deploan & $\begin{array}{c}0.03 \\
(-0.30)\end{array}$ & $\begin{array}{c}0.06 \\
(-1.30)\end{array}$ & $\begin{array}{r}0.03 \\
(-1.19)\end{array}$ & loansale & $\begin{array}{c}1.45 \\
(-14.27)\end{array}$ & $\begin{array}{c}0.7 \\
(-11.59)\end{array}$ & $\begin{array}{r}-0.74 \\
(-0.87)\end{array}$ \\
\hline loangrowth & $\begin{array}{c}-1.7 \\
(-7.30)\end{array}$ & $\begin{array}{c}3.46 \\
(-20.33)\end{array}$ & $\begin{array}{c}5.16 \\
(-10.61)\end{array}$ & brokdep & $\begin{array}{c}19.19 \\
(-18.59)\end{array}$ & $\begin{array}{c}5.46 \\
(-11.79)\end{array}$ & $\begin{array}{c}-13.73 \\
(-12.50)\end{array}$ *** \\
\hline lossallow & $\begin{array}{c}2.91 \\
(-2.09)\end{array}$ & $\begin{array}{c}1.4 \\
(-0.86)\end{array}$ & $\begin{array}{c}-1.51 \\
(12.32) * * *\end{array}$ & interbank & $\begin{array}{c}6.36 \\
(-12.96)\end{array}$ & $\begin{array}{c}1.84 \\
(-8.02)\end{array}$ & $\begin{array}{c}-4.52 \\
(-5.91) * * *\end{array}$ \\
\hline chargeoff & $\begin{array}{c}1.15 \\
(-1.32)\end{array}$ & $\begin{array}{c}0.23 \\
(-0.57)\end{array}$ & $\begin{array}{c}-0.92 \\
(11.86) * * *\end{array}$ & non_income & $\begin{array}{c}-6.98 \\
(-137.39)\end{array}$ & $\begin{array}{c}10.41 \\
(-20.34)\end{array}$ & $\begin{array}{c}17.39 \\
(-2.16) * *\end{array}$ \\
\hline pastdue & $\begin{array}{c}9.82 \\
(-6.40)\end{array}$ & $\begin{array}{c}2.37 \\
(-2.41)\end{array}$ & $\begin{array}{c}-7.45 \\
(19.80) * * *\end{array}$ & cash & $\begin{array}{c}4.81 \\
(-5.31)\end{array}$ & $\begin{array}{c}5.86 \\
(-7.26)\end{array}$ & $\begin{array}{c}1.05 \\
(-3.27) * * *\end{array}$ \\
\hline foreclose & $\begin{array}{c}2.03 \\
(-2.75)\end{array}$ & $\begin{array}{c}0.37 \\
(-0.76)\end{array}$ & $\begin{array}{c}-1.66 \\
(10.27) * * *\end{array}$ & loan_ast & $\begin{array}{c}75.99 \\
(-11.07)\end{array}$ & $\begin{array}{c}66.94 \\
(-17.08)\end{array}$ & $\begin{array}{c}-9.06 \\
(-13.39) \\
* * *\end{array}$ \\
\hline Capital & $\begin{array}{c}6.83 \\
(-3.09)\end{array}$ & $\begin{array}{c}11.85 \\
(-7.87)\end{array}$ & $\begin{array}{c}5.02 \\
(-24.92) * * *\end{array}$ & insureddep & $\begin{array}{c}77.3 \\
(-13.86)\end{array}$ & $\begin{array}{c}76.22 \\
(-14.86)\end{array}$ & $\begin{array}{r}-1.08 \\
(-1.31)\end{array}$ \\
\hline tier1 & $\begin{array}{c}8.32 \\
(-4.08)\end{array}$ & $\begin{array}{c}19.62 \\
(-77.81)\end{array}$ & $\begin{array}{c}11.3 \\
(-12.44)\end{array}$ & & & & \\
\hline
\end{tabular}

Note. We obtained the results by using the cross-sectional data of 2008Q4. Failure dummy variable defined as banks that failed in 2009-2010. We reported the mean of explanatory variables for surviving and failed banks in the first two columns. The standard deviations are in the parenthesis. We also present the difference in mean and the t-statistic in the third column which tests the mean difference of both sample banks. $*$,** and *** significant at the $10 \%, 5 \%$ and $1 \%$ level, variables are described in Table 1 .

The results for each of the tobit models 1 through 5, excluding model 4, are in Appendix Tables A1 through A4 respectively. Results for model 4, discussed here, are given in Table 4. Model 4 appears to be the superior model as each and every variable is significant with an alpha level of at least five percent when using data from the first quarter of 2005. The likelihood ratio (LR) chi-square is very high peaking at 1102.55 in 2007 based on 2005 Quarter 1 . The probability >Chi-square is significant at greater than 0.0000 across all time periods. The log likelihood is in the range of $-129,197$ to $-132,924$ during the entire period. The pseudo R-square is better than the other four models varying from 0.0035 to 0.0041 .

Table 4a. Tobit regression results: Model 4 Panel A (zero equity in 2010)

\begin{tabular}{|c|c|c|c|c|c|c|c|c|c|}
\hline & dependent & 2010 & & 2010 & & 2010 & & 2010 & \\
\hline & independent & 2005Q1 & & 2006Q1 & & 2007Q1 & & 2008Q1 & \\
\hline \multirow[t]{2}{*}{1} & realloan & $-13,666$ & & $-16,840$ & & $-19,529$ & & $-20,412$ & \\
\hline & & $(-10.32)$ & $* * *$ & $(-10.11)$ & $* * *$ & $(-10.39)$ & $* * *$ & $(-9.52)$ & $* * *$ \\
\hline \multirow[t]{2}{*}{2} & capital & 12,967 & & 15,654 & & 14,686 & & 11,812 & \\
\hline & & (4.09) & $* * *$ & $(4.34)$ & $* * *$ & $(3.85)$ & $* * *$ & $(2.75)$ & $* * *$ \\
\hline \multirow[t]{2}{*}{3} & size & 437,080 & & 546,857 & & 603,490 & & 659,423 & \\
\hline & & (31.23) & $* * *$ & $(30.58)$ & $* * *$ & (29.70) & $* * *$ & (29.69) & $* * *$ \\
\hline
\end{tabular}




\begin{tabular}{|c|c|c|c|c|c|c|c|c|c|}
\hline \multirow[t]{2}{*}{4} & \multirow[t]{2}{*}{ roe } & $-3,357$ & & \multicolumn{2}{|l|}{$-2,624$} & \multicolumn{2}{|l|}{$-3,353$} & \multicolumn{2}{|l|}{$-2,810$} \\
\hline & & $(-2.15)$ & $* *$ & $(-1.61)$ & & $(-1.74)$ & $*$ & $(-1.84)$ & $*$ \\
\hline \multirow[t]{2}{*}{5} & lossallow & $-34,024$ & & $-26,046$ & & $-15,687$ & & $-11,419$ & \\
\hline & & $(-2.34)$ & $* *$ & $(-1.45)$ & & $(-0.95)$ & & $(-0.63)$ & \\
\hline \multirow[t]{2}{*}{6} & pastdue & 35,303 & & 46,708 & & 40,746 & & $-3,252$ & \\
\hline & & $(2.92)$ & $* * *$ & (2.95) & $* * *$ & (2.53) & $* *$ & $(-0.27)$ & \\
\hline \multirow[t]{2}{*}{7} & loan_ast & $-10,718$ & & $-14,200$ & & $-11,040$ & & $-20,423$ & \\
\hline & & $(-4.61)$ & $* * *$ & $(-4.88)$ & $* * *$ & $(-3.52)$ & $* * *$ & $(-5.68)$ & $* * *$ \\
\hline \multirow[t]{2}{*}{8} & MBS & $-5,275$ & & $-6,259$ & & $-8,505$ & & $-8,450$ & \\
\hline & & $(-2.60)$ & $* * *$ & $(-2.17)$ & $* *$ & $(-2.43)$ & $* *$ & $(-2.34)$ & $* *$ \\
\hline \multirow[t]{2}{*}{9} & debt_sec & $-16,182$ & & $-20,780$ & & $-18,326$ & & $-27,909$ & \\
\hline & & $(-7.41)$ & $* * *$ & $(-7.46)$ & $* * *$ & $(-5.87)$ & $* * *$ & $(-7.56)$ & $* * *$ \\
\hline \multirow[t]{2}{*}{10} & loansale & $-14,150$ & & $-8,680$ & & $-1,903$ & & 772 & \\
\hline & & $(-2.83)$ & $* * *$ & $(-1.84)$ & $*$ & $(-0.97)$ & & $(0.51)$ & \\
\hline \multirow[t]{2}{*}{11} & insureddep & 2,835 & & 6,471 & & 6,410 & & 5,043 & \\
\hline & & (2.39) & $* *$ & $(4.40)$ & $* * *$ & $(3.79)$ & $* * *$ & $(2.63)$ & $* * *$ \\
\hline \multirow[t]{2}{*}{12} & _cons & $-3,658,909$ & & $-4,835,800$ & & $-5,676,325$ & & $-5,329,084$ & \\
\hline & & $(-13.89)$ & $* * *$ & $(-14.62)$ & $* * *$ & $(-15.50)$ & $* * *$ & $(-12.68)$ & $* * *$ \\
\hline \multicolumn{10}{|c|}{ Model Statistics } \\
\hline & observations & 8,529 & & 8,358 & & 8,226 & & 8,181 & \\
\hline & Censored & 136 & & 142 & & 146 & & 151 & \\
\hline & Uncensored & 8,393 & & 8,216 & & 8,080 & & 8,030 & \\
\hline & LR chi2 & $1,086.4$ & & $1,010.3$ & & 960.2 & & 999.2 & \\
\hline & Prob $>$ chi 2 & 0.0000 & & 0.0000 & & 0.0000 & & 0.0000 & \\
\hline & Log likelihood & $-131,015$ & & $-130,319$ & & $-129,164$ & & $-129,197$ & \\
\hline & Pseudo R2 & 0.0041 & & 0.0039 & & 0.0037 & & 0.0039 & \\
\hline
\end{tabular}

Table 4b. Tobit regression results: Model 4 Panel B (zero equity in 2009)

\begin{tabular}{|c|c|c|c|c|c|c|c|c|c|}
\hline \multirow{3}{*}{1} & \multirow{2}{*}{$\begin{array}{l}\text { dependent } \\
\text { independent }\end{array}$} & \multicolumn{2}{|l|}{2009} & \multicolumn{2}{|l|}{2009} & \multicolumn{2}{|l|}{2009} & \multicolumn{2}{|l|}{2009} \\
\hline & & 2005Q1 & & 2006Q1 & & 2007Q1 & & 2008Q1 & \\
\hline & realloan & $-13,864$ & & $-16,960$ & & $-19,640$ & & $-20,114$ & \\
\hline & & $(-10.48)$ & $* * *$ & $(-10.19)$ & $* * *$ & $(-10.46)$ & $* * *$ & $(-9.39)$ & $* * *$ \\
\hline \multirow[t]{2}{*}{2} & capital & 13,094 & & 16,034 & & 14,891 & & 12,272 & \\
\hline & & (4.14) & $* * *$ & $(4.46)$ & $* * *$ & (3.90) & $* * *$ & $(2.85)$ & $* * *$ \\
\hline \multirow[t]{2}{*}{3} & size & 431,777 & & 538,532 & & 594,031 & & 649,704 & \\
\hline & & (30.86) & $* * *$ & (30.13) & $* * *$ & (29.24) & $* * *$ & (29.25) & $* * *$ \\
\hline \multirow[t]{2}{*}{4} & roe & $-3,392$ & & $-2,723$ & & $-3,319$ & & $-2,153$ & \\
\hline & & $(-2.18)$ & $* *$ & $(-1.67)$ & $*$ & $(-1.73)$ & $*$ & $(-1.40)$ & \\
\hline \multirow[t]{2}{*}{5} & lossallow & $-33,003$ & & $-24,550$ & & $-14,926$ & & $-8,883$ & \\
\hline & & $(-2.27)$ & $* *$ & $(-1.37)$ & & $(-0.91)$ & & $(-0.49)$ & \\
\hline \multirow[t]{2}{*}{6} & pastdue & 36,183 & & 47,947 & & 37,698 & & $-16,944$ & \\
\hline & & $(3.00)$ & $* * *$ & $(3.04)$ & $* * *$ & $(2.34)$ & $* *$ & $(-1.36)$ & \\
\hline \multirow[t]{2}{*}{7} & loan_ast & $-10,918$ & & $-13,659$ & & $-10,642$ & & $-19,675$ & \\
\hline & & $(-4.70)$ & $* * *$ & $(-4.71)$ & $* * *$ & $(-3.40)$ & $* * *$ & $(-5.47)$ & $* * *$ \\
\hline \multirow[t]{2}{*}{8} & MBS & $-4,772$ & & $-5,549$ & & $-8,005$ & & $-8,369$ & \\
\hline & & $(-2.35)$ & $* *$ & $(-1.92)$ & $*$ & $(-2.29)$ & $* *$ & $(-2.32)$ & $* *$ \\
\hline \multirow[t]{2}{*}{9} & debt_sec & $-17,049$ & & $-21,086$ & & $-18,875$ & & $-28,444$ & \\
\hline & & $(-7.82)$ & $* * *$ & $(-7.58)$ & $* * *$ & $(-6.05)$ & $* * *$ & $(-7.70)$ & $* * *$ \\
\hline \multirow[t]{2}{*}{10} & loansale & $-13,865$ & & $-8,602$ & & $-2,033$ & & 798 & \\
\hline & & $(-2.78)$ & $* * *$ & $(-1.82)$ & $*$ & $(-1.03)$ & & $(0.53)$ & \\
\hline \multirow[t]{2}{*}{11} & insureddep & 2,764 & & 6,145 & & 6,227 & & 4,804 & \\
\hline & & (2.33) & $* *$ & (4.19) & $* * *$ & (3.68) & $* * *$ & $(2.51)$ & $* *$ \\
\hline \multirow[t]{2}{*}{12} & _cons & $-3,555,618$ & & $-4,744,254$ & & $-5,559,425$ & & $-5,231,439$ & \\
\hline & & $(-13.51)$ & $* * *$ & $(-14.36)$ & $* * *$ & $(-15.18)$ & $* * *$ & $(-12.45)$ & $* * *$ \\
\hline
\end{tabular}




\begin{tabular}{lrrrr}
\hline & \multicolumn{4}{c}{ Model Statistics } \\
observations & 8,529 & 8,358 & 8,226 & 8,181 \\
Censored & 125 & 127 & 134 & 137 \\
Uncensored & 8,404 & 8,231 & 8,092 & 8,044 \\
LR chi2 & $1,070.4$ & 988.3 & 936.9 & 977.8 \\
Prob > chi2 & 0.0000 & 0.0000 & 0.0000 & 0.0000 \\
Log likelihood & $-131,180$ & $-130,549$ & $-129,353$ & $-129,415$ \\
Pseudo R2 & 0.0041 & 0.0038 & 0.0036 & 0.0038 \\
\hline
\end{tabular}

Table 4c. Tobit regression results: Model 4 Panel C (zero equity in 2008)

\begin{tabular}{|c|c|c|c|c|c|c|c|c|}
\hline dependent & 2008 & & 2008 & & 2008 & & 2008 & \\
\hline independent & 2005Q1 & & 2006Q1 & & 2007Q1 & & 2008Q1 & \\
\hline \multirow[t]{2}{*}{ realloan } & $-13,184$ & & $-16,100$ & & $-18,405$ & & $-18,949$ & \\
\hline & $(-10.05)$ & $* * *$ & $(-9.88)$ & $* * *$ & $(-9.98)$ & $* * *$ & $(-8.97)$ & $* * *$ \\
\hline \multirow[t]{2}{*}{ capital } & 13,123 & & 15,751 & & 14,652 & & 11,869 & \\
\hline & $(4.18)$ & $* * *$ & $(4.47)$ & $* * *$ & $(3.90)$ & $* * *$ & $(2.80)$ & $* * *$ \\
\hline \multirow[t]{2}{*}{ size } & 433,451 & & 520,994 & & 579,184 & & 638,148 & \\
\hline & $(31.25)$ & $* * *$ & (29.76) & $* * *$ & (28.99) & $* * *$ & (29.12) & $* * *$ \\
\hline \multirow[t]{2}{*}{ roe } & $-3,333$ & & $-2,575$ & & $-3,265$ & & $-1,741$ & \\
\hline & $(-2.16)$ & $* *$ & $(-1.61)$ & & $(-1.73)$ & $*$ & $(-1.12)$ & \\
\hline \multirow[t]{2}{*}{ lossallow } & $-31,699$ & & $-22,224$ & & $-13,450$ & & $-8,616$ & \\
\hline & $(-2.20)$ & $* *$ & $(-1.26)$ & & $(-0.83)$ & & $(-0.48)$ & \\
\hline \multirow[t]{2}{*}{ pastdue } & 35,765 & & 45,481 & & 41,054 & & $-2,357$ & \\
\hline & (2.99) & $* * *$ & $(2.95)$ & $* * *$ & $(2.60)$ & $* * *$ & $(-0.19)$ & \\
\hline \multirow[t]{2}{*}{ loan_ast } & $-10,833$ & & $-13,230$ & & $-10,418$ & & $-19,829$ & \\
\hline & $(-4.70)$ & $* * *$ & $(-4.65)$ & $* * *$ & $(-3.38)$ & $* * *$ & $(-5.59)$ & $* * *$ \\
\hline \multirow[t]{2}{*}{ MBS } & $-4,741$ & & $-5,165$ & & $-7,541$ & & $-7,536$ & \\
\hline & $(-2.36)$ & $* *$ & $(-1.83)$ & $*$ & $(-2.19)$ & $* *$ & $(-2.12)$ & $* *$ \\
\hline \multirow[t]{2}{*}{ debt_sec } & $-16,716$ & & $-20,510$ & & $-18,222$ & & $-28,159$ & \\
\hline & $(-7.72)$ & $* * *$ & $(-7.53)$ & $* * *$ & $(-5.94)$ & $* * *$ & $(-7.73)$ & $* * *$ \\
\hline \multirow[t]{2}{*}{ loansale } & $-12,413$ & & $-7,469$ & & $-1,729$ & & 803 & \\
\hline & $(-2.52)$ & $* *$ & $(-1.62)$ & & $(-0.89)$ & & $(0.54)$ & \\
\hline \multirow[t]{2}{*}{ insureddep } & 2,539 & & 5,247 & & 5,102 & & 3,953 & \\
\hline & (2.16) & $* *$ & $(3.66)$ & $* * *$ & $(3.07)$ & $* * *$ & $(2.09)$ & $* *$ \\
\hline \multirow[t]{2}{*}{ _cons } & $-3,590,822$ & & $-4,534,848$ & & $-5,371,054$ & & $-5,089,209$ & \\
\hline & $(-13.76)$ & $* * *$ & $(-14.01)$ & $* * *$ & $(-14.92)$ & $* * *$ & $(-12.28)$ & $* * *$ \\
\hline \multicolumn{9}{|c|}{ Model Statistics } \\
\hline observations & 8,529 & & 8,358 & & 8,226 & & 8,181 & \\
\hline Censored & 21 & & 23 & & 25 & & 23 & \\
\hline Uncensored & 8,508 & & 8,335 & & 8,201 & & 8,158 & \\
\hline LR chi2 & $1,092.0$ & & 973.3 & & 928.0 & & 971.6 & \\
\hline Prob > chi2 & 0.0000 & & 0.0000 & & 0.0000 & & 0.0000 & \\
\hline Log likelihood & $-132,669$ & & $-131,961$ & & $-130,889$ & & $-131,067$ & \\
\hline Pseudo R2 & 0.0041 & & 0.0037 & & 0.0035 & & 0.0037 & \\
\hline
\end{tabular}

Table 4d. Tobit regression results: Model 4 Panel D (zero equity in 2007)

\begin{tabular}{|c|c|c|c|c|c|c|c|}
\hline & dependent & 2007 & & 2007 & & 2007 & \\
\hline & independent & 2005Q1 & & 2006Q1 & & 2007Q1 & \\
\hline \multirow[t]{2}{*}{1} & realloan & $-13,107$ & & $-15,840$ & & $-18,123$ & \\
\hline & & $(-10.01)$ & $* * *$ & $(-9.62)$ & $* * *$ & $(-9.77)$ & $* * *$ \\
\hline \multirow[t]{2}{*}{2} & capital & 13,208 & & 16,185 & & 15,043 & \\
\hline & & $(4.22)$ & $* * *$ & $(4.55)$ & $* * *$ & (3.98) & $* * *$ \\
\hline \multirow[t]{2}{*}{3} & size & 435,364 & & 542,596 & & 599,707 & \\
\hline & & (31.44) & $* * *$ & (30.68) & $* * *$ & (29.87) & $* * *$ \\
\hline
\end{tabular}




\begin{tabular}{|c|c|c|c|c|c|c|c|}
\hline 4 & roe & $\begin{array}{l}-3,424 \\
(-2.22)\end{array}$ & $* *$ & $\begin{array}{l}-2,656 \\
(-1.64)\end{array}$ & & $\begin{array}{l}-3,095 \\
(-1.62)\end{array}$ & \\
\hline 5 & lossallow & $\begin{array}{r}-31,743 \\
(-2.20)\end{array}$ & $* *$ & $\begin{array}{r}-23,499 \\
(-1.32)\end{array}$ & & $\begin{array}{r}-13,984 \\
(-0.86)\end{array}$ & \\
\hline 6 & pastdue & $\begin{array}{r}35,926 \\
(3.01)\end{array}$ & $* * *$ & $\begin{array}{r}50,372 \\
(3.24)\end{array}$ & $* * *$ & $\begin{array}{r}45,842 \\
(2.89)\end{array}$ & $* * *$ \\
\hline 7 & loan_ast & $\begin{array}{r}-10,928 \\
(-4.75)\end{array}$ & $* * *$ & $\begin{array}{r}-13,989 \\
(-4.87)\end{array}$ & $* * *$ & $\begin{array}{r}-11,116 \\
(-3.59)\end{array}$ & $* * *$ \\
\hline 8 & MBS & $\begin{array}{l}-4,755 \\
(-2.37)\end{array}$ & $* *$ & $\begin{array}{l}-5,676 \\
(-1.99)\end{array}$ & $* *$ & $\begin{array}{l}-8,088 \\
(-2.34)\end{array}$ & $* *$ \\
\hline 9 & debt_sec & $\begin{array}{r}-16,851 \\
(-7.80)\end{array}$ & $* * *$ & $\begin{array}{r}-21,008 \\
(-7.63)\end{array}$ & $* * *$ & $\begin{array}{r}-18,695 \\
(-6.05)\end{array}$ & $* * *$ \\
\hline 10 & loansale & $\begin{array}{r}-11,599 \\
(-2.36)\end{array}$ & $* *$ & $\begin{array}{l}-7,561 \\
(-1.62)\end{array}$ & & $\begin{array}{l}-1,724 \\
(-0.89)\end{array}$ & \\
\hline 11 & insureddep & $\begin{array}{l}2,620 \\
(2.23)\end{array}$ & $* *$ & $\begin{array}{l}5,451 \\
(3.76)\end{array}$ & $* * *$ & $\begin{array}{l}5,393 \\
(3.23)\end{array}$ & $* * *$ \\
\hline 12 & _cons & $\begin{array}{r}-3,612,255 \\
(-13.87) \\
\end{array}$ & $* * *$ & $\begin{array}{r}-4,756,251 \\
(-14.55) \\
\end{array}$ & $* * *$ & $\begin{array}{r}-5,596,531 \\
(-15.46) \\
\end{array}$ & $* * *$ \\
\hline \multicolumn{8}{|c|}{ Model Statistics } \\
\hline & observations & 8,529 & & 8,358 & & 8,226 & \\
\hline & Censored & 3 & & 3 & & 2 & \\
\hline & Uncensored & 8,526 & & 8,355 & & 8,224 & \\
\hline & LR chi2 & $1,102.6$ & & $1,023.3$ & & 974.8 & \\
\hline & Prob $>$ chi 2 & 0.0000 & & 0.0000 & & 0.0000 & \\
\hline & Log likelihood & $-132,924$ & & $-132,351$ & & $-131,287$ & \\
\hline & Pseudo R2 & 0.0041 & & 0.0039 & & 0.0037 & \\
\hline
\end{tabular}

The set of factors in model 4 explaining equity capital includes real estate loans to assets, equity capital to total assets, $\log$ of total assets, return on equity, loan loss allowance to total loans, non-performing loans to total assets, total loans to total assets, mortgage-backed securities to total assets, total short-term debt securities to total assets, net gains on sales of loans to total non-interest income, and insured deposits to total deposits. The constant (in all of the models) is negative, very large ( $\$ 3$ million and up), and always significant. It is interesting to note that roe, lossallow, pastdue, and loansale all become less significant after 2005 even as the banks come closer to failure.

Similar to the univariate analysis (Tables 2 and 3), the tobit analysis (Tables A1 through A4 and Table 4) show an unexpected negative effect on equity with increased exposure to multi-family real estate loans, total loans to total assets, and real estate loans to total loans. However, tobit analysis shows a very strong and very large positive association between the size of the bank in terms of total assets and the expected equity value of the bank.

\section{Conclusions}

This paper studies US banks whose equity capital evaporated resulting in their demise during the financial crisis and economic recession of 2008 to 2010. The univariate t-test method is used to detect mean differences for 29 independent financial variables between censored banks (zero equity capital) and noncensored banks (positive equity capital). The tobit regression analysis indicates that realloan, capital, size, roe, lossallow, pastdue, loan_ast, MBS, debt_sec, loansale, and insureddep are the most significant in determining the amount of equity banks were able to maintain during the crisis. Comparing failed to surviving banks we discover a great disparity in performance. The operations of banks undergoing reductions in equity capital were far different in terms of riskiness of assets, capital structure, liquidity, and profitability. In particular, banks with plummeting equity capital had a loan portfolio tilted towards real estate and construction, higher levels of debt on the balance sheet, lower cash levels, and operating losses.

Managers as well as regulators need to take into consideration the danger that banks can pass into when taking on riskier loans and overexposing their loan portfolio to 1 or 2 industries. The result of such decisions leads to a low quality loan portfolio generating losses that ripple into overall operating losses reducing the amount of the equity capital safety cushion. This situation can lead to bank failure.

\section{References}

Acharya, V. V., \& Viswanathan, S. (2011). Leverage, moral hazard, and liquidity. Journal of Finance, 66, 99-138. 
http://dx.doi.org/10.1111/j.1540-6261.2010.01627.x

Brunnermeier, M. K., \& Pedersen, L. H. (2009). Market liquidity and funding liquidity. Review of Financial Studies, 22, 2201-2238. http://dx.doi.org/10.1093/rfs/hhn098

Cihak, M., \& Schaeck, K. (2010). How well do aggregate prudential ratios identify banking system problems? Journal of Financial Stability, 6, 130-144. http://dx.doi.org/10.1016/j.jfs.2010.03.001

Cole, R. A., \& White, L. J. (2012). Déjà vu all over again: The causes of US commercial bank failures this time around. Journal of Financial Services Research, 42, 5-29. http://dx.doi.org/10.1007/s10693-011-0116-9

Cox, R. A. K., \& Wang, G. (2014). Predicting the US bank failure: A discriminant analysis. Economic Analysis and Policy, 44, 202-211. http://dx.doi.org/10.1016/j.eap.2014.06.002

Foster, G., \& Kalenkoski, C. M. (2013). Tobit or OLS? An empirical evaluation under different diary window lengths. Applied Economics, 45, 2994-3010. http://dx.doi.org/10.1080/00036846.2012.690852

Gonzalez-Hermosillo, B. (1999). Determinants of ex-ante banking system distress: A macro-micro empirical exploration of some recent episodes. IMF Working Paper, 99/33. http://dx.doi.org/10.5089/9781451845167.001

Ho, P. H., Huang, C. W., Lin, C. Y., \& Yen, J. F. (2016). CEO overconfidence and financial crisis: Evidence from bank lending and leverage. Journal of Financial Economics, 120, 194-209. http://dx.doi.org/10.1016/j.jfineco.2015.04.007

Hutchison, D. E., \& Cox, R. A. K. (n. d.). The causal relationship between bank capital and profitability. Annals of Financial Economics, 20, 56-62. http://dx.doi.org/10.1142/s2010495207500029

James, C. (1991). The losses realized in bank failures. Journal of Finance, 45, 1223-1242. http://dx.doi.org/10.1111/j.1540-6261.1991.tb04616.x

Mare, D. S. (2015). Contribution of macroeconomic factors to the prediction of small bank failures. Journal of International Financial Markets, Institutions and Money, 39, 25-39. http://dx.doi.org/10.1016/j.intfin.2015.05.005

McDonald, J. F., \& Moffitt, R. A. (1980). The uses of tobit analysis. Review of Economics and Statistics, 62, 318-321. http://dx.doi.org/10.2307/1924766

Shleifer, A., \& Vishny, R. W. (2010). Unstable Banking. Journal of Financial Economics, 97, 306-318. http://dx.doi.org/10.1016/j.jfineco.2009.10.007

Sinkey Jr., J. F. (1974). The way problem banks perform. The Bankers Magazine, 57, 40-51.

Sinkey Jr., J. F. (1975). A multivariate statistical analysis of the characteristics of problem banks. Journal of Finance, 30, 21-36. http://dx.doi.org/10.1111/j.1540-6261.1975.tb03158.x

Stewart, J. (2013). Tobit or not tobit? Journal of Economic and Social Measurement, 38, 263-290. http://dx.doi.org/10.3233/JEM-130376

Tobit, J. (1958). Estimation of relationships for limited dependent variables. Econometrica, 26, 24-36. http://dx.doi.org/10.2307/1907382

Uzun, H., \& Webb, E. ((2007). Securitization and risk: Empirical evidence on US banks. Journal of Risk Finance, 8, 11-23. http://dx.doi.org/10.1108/15265940710721046

Wagner, W. (2007). The liquidity of bank assets and banking stability. Journal of Banking and Finance, 31, 121-139. http://dx.doi.org/10.1016/j.jbankfin.2005.07.019

Walter, J. R. (2004). Closing troubled banks: How the process works. Federal Reserve Bank of Richmond Economic Quarterly, 90(1), 51-68. http://dx.doi.org/10.2307/1053138 


\section{Appendix A}

Table A1. Tobit regression results: Model 1

\begin{tabular}{|c|c|c|c|c|c|c|c|c|c|}
\hline \multicolumn{10}{|c|}{ Tobit regression results: Model 1 Panel A (zero equity in 2010) } \\
\hline & \multirow{2}{*}{$\begin{array}{l}\text { dependent } \\
\text { independent }\end{array}$} & \multicolumn{2}{|l|}{2010} & \multicolumn{2}{|l|}{2010} & \multicolumn{2}{|l|}{2010} & \multicolumn{2}{|l|}{2010} \\
\hline & & 2005Q1 & & 2006Q1 & & 2007Q1 & & 2008Q1 & \\
\hline \multirow[t]{2}{*}{1} & realloan & $-10,476$ & & $-13,737$ & & $-15,982$ & & $-19,205$ & \\
\hline & & $(-9.98)$ & $* * *$ & $(-10.31)$ & $* * *$ & $(-10.52)$ & $* * *$ & $(-11.31)$ & $* * *$ \\
\hline \multirow[t]{2}{*}{2} & idloan & 10,322 & & 10,032 & & 12,702 & & 13,071 & \\
\hline & & $(3.88)$ & $* * *$ & $(2.94)$ & $* * *$ & $(3.22)$ & $* * *$ & $(2.88)$ & $* * *$ \\
\hline \multirow[t]{2}{*}{3} & deploan & 108,801 & & 77,480 & & 73,479 & & 87,849 & \\
\hline & & $(6.29)$ & $* * *$ & $(4.63)$ & $* * *$ & $(3.27)$ & $* * *$ & (3.07) & $* * *$ \\
\hline \multirow[t]{2}{*}{4} & loangrowth & -136.5868 & & 1.3205 & & -7.3645 & & 2.2262 & \\
\hline & & $(-0.38)$ & & $(-0.15)$ & & $(-0.43)$ & & $(0.02)$ & \\
\hline \multirow[t]{2}{*}{5} & capital & 10,305 & & 14,286 & & 11,629 & & 13,742 & \\
\hline & & (2.89) & $* * *$ & $(3.63)$ & $* * *$ & $(2.93)$ & $* * *$ & $(3.02)$ & $* * *$ \\
\hline \multirow[t]{2}{*}{6} & size & 394,417 & & 479,896 & & 544,773 & & 629,038 & \\
\hline & & $(28.51)$ & $* * *$ & $(27.41)$ & $* * *$ & $(27.20)$ & $* * *$ & (28.08) & $* * *$ \\
\hline \multirow[t]{2}{*}{7} & roa & $-34,724$ & & 5,066 & & $-45,220$ & & $-47,127$ & \\
\hline & & $(-2.93)$ & $* * *$ & $(-0.50)$ & & $(-2.45)$ & $* *$ & $(-2.87)$ & $* * *$ \\
\hline \multirow[t]{10}{*}{8} & \multirow{2}{*}{ constant } & $-4,227,085$ & & $-5,162,766$ & & $-5,790,735$ & & $-6,689,067$ & \\
\hline & & $(-24.91)$ & $* * *$ & $(-23.99)$ & $* * *$ & $(-23.78)$ & $* * *$ & $(-24.20)$ & $* * *$ \\
\hline & \multicolumn{9}{|c|}{ Model Statistics } \\
\hline & observations & 7,719 & & 7,580 & & 7,480 & & 7,434 & \\
\hline & Censored & 119 & & 124 & & 130 & & 133 & \\
\hline & Uncensored & 7,600 & & 7,456 & & 7,350 & & 7,301 & \\
\hline & LR chi2 & 886.31 & & 799.46 & & 774.21 & & 826.9 & \\
\hline & Prob > chi2 & 0.0000 & & 0.0000 & & 0.0000 & & 0.0000 & \\
\hline & Log likelihood & $-118,835$ & & $-118,416$ & & $-117,635$ & & $-117,722$ & \\
\hline & Pseudo R2 & 0.0037 & & 0.0034 & & 0.0033 & & 0.0035 & \\
\hline & & Tobit regress & on resu & Model 1 Pane & $\mathrm{B}(\mathrm{ze}$ & quity in 2009) & & & \\
\hline & dependent & 2009 & & 2009 & & 2009 & & 2009 & \\
\hline & independent & 2005Q1 & & 2006Q1 & & 2007Q1 & & 2008Q1 & \\
\hline 1 & realloan & $-10,370$ & & $-13,491$ & & $-15,651$ & & $-18,537$ & \\
\hline & & $(-9.88)$ & $* * *$ & $(-10.14)$ & $* * *$ & $(-10.31)$ & $* * *$ & $(-10.93)$ & $* * *$ \\
\hline 2 & idloan & 10,540 & & 10,299 & & 13,025 & & 13,298 & \\
\hline & & $(3.96)$ & $* * *$ & $(3.02)$ & $* * *$ & $(3.31)$ & $* * *$ & (2.93) & $* * *$ \\
\hline 3 & deploan & 108,718 & & 77,619 & & 73,840 & & 89,633 & \\
\hline & & $(6.29)$ & $* * *$ & $(0.00)$ & & (3.29) & $* * *$ & (3.14) & $* * *$ \\
\hline 4 & loangrowth & -166.6617 & & -1.2300 & & -7.3554 & & 3.6656 & \\
\hline & & $(-0.47)$ & & (0.89) & & $(-0.43)$ & & $(0.04)$ & \\
\hline 5 & capital & 10,749 & & 14,262 & & 11,659 & & 14,917 & \\
\hline & & (3.02) & $* * *$ & $(0.00)$ & & $(2.93)$ & $* * *$ & $(3.28)$ & $* * *$ \\
\hline 6 & size & 391,966 & & 476,757 & & 539,107 & & 622,745 & \\
\hline & & (28.34) & $* * *$ & $(0.00)$ & & $(26.92)$ & $* * *$ & $(27.80)$ & $* * *$ \\
\hline 7 & roa & $-35,776$ & & $-4,735$ & & $-45,001$ & & $-38,037$ & \\
\hline & & $(-3.02)$ & $* * *$ & $(0.64)$ & & $(-2.43)$ & $* *$ & $(-2.30)$ & $* *$ \\
\hline 8 & constant & $-4,205,584$ & & $-5,135,496$ & & $-5,737,946$ & & $-6,662,273$ & \\
\hline & & $(-24.80)$ & $* * *$ & $(0.00)$ & & $(-23.56)$ & $* * *$ & $(-24.11)$ & $* * *$ \\
\hline & & & & Iodel Statistic & & & & & \\
\hline & observations & 7,719 & & 7,580 & & 7,480 & & 7,434 & \\
\hline & Censored & 104 & & 110 & & 118 & & 118 & \\
\hline & Uncensored & 7,615 & & 7,470 & & 7,362 & & 7,316 & \\
\hline & LR chi2 & 877.78 & & 790.58 & & 759.17 & & 811.27 & \\
\hline & Prob $>$ chi 2 & 0.0000 & & 0.0000 & & 0.0000 & & 0.0000 & \\
\hline & Log likelihood & $-119,055$ & & $-118,626$ & & $-117,820$ & & $-117,953$ & \\
\hline & Pseudo R2 & 0.0037 & & 0.0033 & & 0.0032 & & 0.0034 & \\
\hline
\end{tabular}




\begin{tabular}{|c|c|c|c|c|c|c|c|c|c|}
\hline \multicolumn{10}{|c|}{ Tobit regression results: Model 1 Panel C (zero equity in 2008) } \\
\hline & dependent & 2008 & & 2008 & & 2008 & & 2008 & \\
\hline & independent & 2005Q1 & & 2006Q1 & & 2007Q1 & & 2008Q1 & \\
\hline \multirow[t]{2}{*}{1} & realloan & $-9,807$ & & $-12,736$ & & $-14,720$ & & $-17,569$ & \\
\hline & & $(-9.43)$ & $* * *$ & $(-9.66)$ & $* * *$ & $(-9.80)$ & $* * *$ & $(-10.46)$ & $* * *$ \\
\hline \multirow[t]{2}{*}{2} & idloan & 9,936 & & 9,534 & & 12,137 & & 12,474 & \\
\hline & & $(3.76)$ & $* * *$ & $(2.81)$ & $* * *$ & $(3.10)$ & $* * *$ & $(2.77)$ & $* * *$ \\
\hline \multirow[t]{2}{*}{3} & deploan & 108,536 & & 76,794 & & 72,759 & & 88,744 & \\
\hline & & $(6.32)$ & $* * *$ & $(4.63)$ & $* * *$ & $(3.27)$ & $* * *$ & $(3.13)$ & $* * *$ \\
\hline \multirow[t]{2}{*}{4} & loangrowth & -140.9246 & & -1.3760 & & -7.4398 & & 1.4038 & \\
\hline & & $(-0.40)$ & & $(-0.16)$ & & $(-0.44)$ & & $(0.01)$ & \\
\hline \multirow[t]{2}{*}{5} & capital & 10,870 & & 14,976 & & 12,461 & & 14,383 & \\
\hline & & $(3.08)$ & $* * *$ & $(3.84)$ & $* * *$ & $(3.17)$ & $* * *$ & $(3.18)$ & $* * *$ \\
\hline \multirow[t]{2}{*}{6} & size & 394,857 & & 480,622 & & 545,664 & & 629,590 & \\
\hline & & (28.78) & $* * *$ & (27.69) & $* * *$ & $(27.51)$ & $* * *$ & (28.39) & $* * *$ \\
\hline \multirow[t]{2}{*}{7} & roa & $-35,634$ & & $-4,830$ & & $-45,461$ & & $-44,005$ & \\
\hline & & $(-3.03)$ & $* * *$ & $(-0.48)$ & & $(-2.48)$ & $* *$ & $(-2.68)$ & $* * *$ \\
\hline \multirow[t]{10}{*}{8} & constant & $-4,248,533$ & & $-5,200,040$ & & $-5,839,780$ & & $-6,747,984$ & \\
\hline & & $(-25.25)$ & $* * *$ & $(-24.37)$ & $* * *$ & $(-24.20)$ & $* * *$ & $(-24.66)$ & $* * *$ \\
\hline & \multicolumn{9}{|c|}{ Model Statistics } \\
\hline & observations & \multicolumn{2}{|l|}{7,719} & \multicolumn{2}{|l|}{7,580} & \multicolumn{2}{|l|}{7,480} & \multicolumn{2}{|l|}{7,434} \\
\hline & Censored & \multicolumn{2}{|l|}{17} & \multicolumn{2}{|l|}{18} & 20 & & 18 & \\
\hline & Uncensored & 7,702 & & 7,562 & & 7,460 & & 7,416 & \\
\hline & LR chi2 & 895.08 & & 807.05 & & 781.71 & & 833.48 & \\
\hline & Prob > chi2 & 0.0000 & & 0.0000 & & 0.0000 & & 0.0000 & \\
\hline & Log likelihood & $-120,304$ & & $-119,969$ & & $-119,260$ & & 119,435 & \\
\hline & Pseudo R2 & 0.0037 & & 0.0034 & & 0.0033 & & 0.0035 & \\
\hline & & Tobit regress & on resu & Model 1 Pane & $\mathrm{D}(\mathrm{ze}$ & quity in 2007) & & & \\
\hline & dependent & 2007 & & 2007 & & 2007 & & & \\
\hline & independent & 2005Q1 & & 2006Q1 & & 2007Q1 & & & \\
\hline 1 & realloan & $-9,702$ & & $-12,569$ & & $-14,482$ & & & \\
\hline & & $(-9.34)$ & $* * *$ & $(-9.55)$ & $* * *$ & $(-9.66)$ & $* * *$ & & \\
\hline 2 & idloan & 9,900 & & 9,448 & & 12,007 & & & \\
\hline & & $(3.75)$ & $* * *$ & $(2.79)$ & $* * *$ & $(3.08)$ & $* * *$ & & \\
\hline 3 & deploan & 108,448 & & 76,679 & & 73,053 & & & \\
\hline & & $(6.33)$ & $* * *$ & $(4.63)$ & $* * *$ & $(3.28)$ & $* * *$ & & \\
\hline 4 & loangrowth & -146.2282 & & -1.4324 & & -7.3611 & & & \\
\hline & & $(-0.41)$ & & $(-0.17)$ & & $(-0.43)$ & & & \\
\hline 5 & capital & 11,071 & & 15,252 & & 12,553 & & & \\
\hline & & (3.14) & $* * *$ & $(3.91)$ & $* * *$ & $(3.20)$ & $* * *$ & & \\
\hline 6 & size & 395,415 & & 481,621 & & 546,230 & & & \\
\hline & & $(4.82)$ & $* * *$ & (27.79) & $* * *$ & $(27.58)$ & $* * *$ & & \\
\hline 7 & roa & $-36,646$ & & $-4,891$ & & $-44,353$ & & & \\
\hline & & $(-3.12)$ & $* * *$ & $(-0.48)$ & & $(-2.42)$ & $* *$ & & \\
\hline 8 & constant & $-4,258,254$ & & $-5,218,745$ & & $-5,854,437$ & & & \\
\hline & & $(-25.34)$ & $* * *$ & $(-24.50)$ & $* * *$ & $(-24.31)$ & $* * *$ & & \\
\hline & & & & Iodel Statisti & & & & & \\
\hline & observations & 7,719 & & 7,580 & & 7,480 & & & \\
\hline & Censored & 2 & & 2 & & 1 & & & \\
\hline & Uncensored & 7,717 & & 7,578 & & 7,479 & & & \\
\hline & LR chi2 & 897.98 & & 810.84 & & 784.51 & & & \\
\hline & Prob > chi 2 & 0.0000 & & 0.0000 & & 0.0000 & & & \\
\hline & Log likelihood & $-120,519$ & & 120,202 & & $-119,540$ & & & \\
\hline & Pseudo R2 & 0.0037 & & 0.0034 & & 0.0033 & & & \\
\hline
\end{tabular}

Note. $\mathrm{t}$-statistics are in the parentheses. *, ** and $* * *$ indicate statistical significance at $10 \%, 5 \%, 1 \%$ level, respectively. All variables are described in Table 1. 
Table A2. Tobit regression results: Model 2

\begin{tabular}{|c|c|c|c|c|c|c|c|c|c|}
\hline \multicolumn{10}{|c|}{ Tobit regression results: Model 2 Panel A (zero equity in 2010) } \\
\hline & \multirow{2}{*}{$\begin{array}{l}\text { dependent } \\
\text { independent }\end{array}$} & \multicolumn{2}{|l|}{2010} & \multicolumn{2}{|l|}{2010} & \multicolumn{2}{|l|}{2010} & \multicolumn{2}{|l|}{2010} \\
\hline & & 2005Q1 & & 2006Q1 & & \multicolumn{2}{|l|}{ 2007Q1 } & \multicolumn{2}{|l|}{ 2008Q1 } \\
\hline \multirow[t]{2}{*}{1} & cons_devlp & $-13,159$ & & $-16,605$ & & $-19,877$ & & $-22,165$ & \\
\hline & & $(-8.98)$ & $* * *$ & $(-9.73)$ & $* * *$ & $(-10.38)$ & $* * *$ & $(-10.08)$ & $* * *$ \\
\hline \multirow[t]{2}{*}{2} & comm_real & $-10,955$ & & $-13,771$ & & $-16,648$ & & $-18,168$ & \\
\hline & & $(-9.13)$ & $* * *$ & $(-8.89)$ & $* * *$ & $(-9.22)$ & $* * *$ & $(-9.05)$ & $* * *$ \\
\hline \multirow[t]{2}{*}{3} & mul_family & $-14,155$ & & $-19,361$ & & $-9,085$ & & $-3,305$ & \\
\hline & & $(-5.04)$ & $* * *$ & $(-5.17)$ & $* * *$ & $(-2.09)$ & $* *$ & $(-0.72)$ & \\
\hline \multirow[t]{2}{*}{4} & sig_family & $-4,134.1380$ & & $-4,744.0000$ & & $-5,125.3680$ & & $-6,029.7460$ & \\
\hline & & $(-3.98)$ & $* * *$ & $(-3.50)$ & $* * *$ & $(-3.23)$ & $* * *$ & $(-3.39)$ & $* * *$ \\
\hline \multirow[t]{2}{*}{5} & ciloan & 8,037 & & 10,131 & & 13,451 & & 14,358 & \\
\hline & & $(3.61)$ & $* * *$ & $(3.59)$ & $* * *$ & $(4.06)$ & $* * *$ & $(3.91)$ & $* * *$ \\
\hline \multirow[t]{2}{*}{6} & capital & 25,933 & & 29,510 & & 35,245 & & 31,569 & \\
\hline & & $(8.72)$ & $* * *$ & $(8.52)$ & $* * *$ & $(9.48)$ & $* * *$ & (7.66) & $* * *$ \\
\hline 7 & size & 415,366 & & 507,585 & & 588,116 & & 624,177 & \\
\hline & & $(30.86)$ & $* * *$ & $(29.75)$ & $* * *$ & $(30.05)$ & $* * *$ & $(29.01)$ & $* * *$ \\
\hline 8 & roa & $-13,322$ & & $-4,638$ & & 6,691 & & $-30,123$ & \\
\hline & & $(-1.29)$ & & $(-0.35)$ & & $(0.42)$ & & $(-2.06)$ & $* *$ \\
\hline 9 & _cons & $-4,445,743$ & & $-5,435,014$ & & $-6,392,030$ & & $-6,689,435$ & \\
\hline & & $(-27.59)$ & $* * *$ & $(-26.43)$ & $* * *$ & $(-26.94)$ & $* * *$ & $(-25.24)$ & $* * *$ \\
\hline & & & & odel Statistics & & & & & \\
\hline & observations & 8,515 & & 8,343 & & 8,217 & & 8,187 & \\
\hline & Censored & 136 & & 142 & & 146 & & 150 & \\
\hline & Uncensored & 8,379 & & 8,201 & & 8,071 & & 8,037 & \\
\hline & LR chi2 & 964.5 & & 905 & & 951.58 & & 888.55 & \\
\hline & Prob > chi 2 & 0.0000 & & 0.0000 & & 0.0000 & & 0.0000 & \\
\hline & Log likelihood & $-130,779$ & & $-129,942$ & & $-129,022$ & & $-129,292$ & \\
\hline & Pseudo R2 & 0.0037 & & 0.0035 & & 0.0037 & & 0.0034 & \\
\hline & & Tobit regressic & n resul & Model 2 Panel & (zerc & ity in 2009) & & & \\
\hline & dependent & 2009 & & 2009 & & 2009 & & 2009 & \\
\hline & independent & 2005Q1 & & 2006Q1 & & 2007Q1 & & 2008Q1 & \\
\hline 1 & cons_devlp & $-13,159$ & & $-16,586$ & & $-19,680$ & & $-21,854$ & \\
\hline & & $(-8.96)$ & $* * *$ & $(-9.70)$ & $* * *$ & $(-10.26)$ & $* * *$ & $(-9.93)$ & $* * *$ \\
\hline 2 & comm_real & $-10,671$ & & $-13,322$ & & $-16,213$ & & $-17,379$ & \\
\hline & & $(-8.90)$ & $* * *$ & $(-8.60)$ & $* * *$ & $(-8.98)$ & $* * *$ & $(-8.66)$ & $* * *$ \\
\hline 3 & mul_family & $-14,745$ & & $-19,632$ & & $-9,438$ & & $-3,487$ & \\
\hline & & $(-5.23)$ & $* * *$ & $(-5.23)$ & $* * *$ & $(-2.16)$ & $* *$ & $(-0.76)$ & \\
\hline 4 & sig_family & $-3,829$ & & $-4,339$ & & $-4,707$ & & $-5,510$ & \\
\hline & & $(0.00)$ & & $(-3.20)$ & $* * *$ & $(-2.96)$ & $* * *$ & $(-3.10)$ & $* * *$ \\
\hline 5 & ciloan & 8,827 & & 11,100 & & 14,415 & & 15,160 & \\
\hline & & $(3.98)$ & $* * *$ & $(3.95)$ & $* * *$ & $(4.35)$ & $* * *$ & $(4.14)$ & $* * *$ \\
\hline 6 & capital & 25,765 & & 29,721 & & 35,167 & & 33,072 & \\
\hline & & (8.63) & $* * *$ & $(8.61)$ & $* * *$ & $(9.45)$ & $* * *$ & $(8.01)$ & $* * *$ \\
\hline 7 & size & 409,605 & & 500,487 & & 579,692 & & 615,826 & \\
\hline & & $(30.42)$ & $* * *$ & $(29.33)$ & $* * *$ & $(29.61)$ & $* * *$ & $(28.60)$ & $* * *$ \\
\hline 8 & roa & $-13,235$ & & $-4,758$ & & 6,584 & & $-19,598$ & \\
\hline & & $(-1.28)$ & & $(-0.36)$ & & $(0.41)$ & & $(-1.33)$ & \\
\hline 9 & _cons & $-4,402,957$ & & $-5,390,737$ & & $-6,330,278$ & & $-6,668,479$ & \\
\hline & & $(-27.32)$ & $* * *$ & $(-26.22)$ & $* * *$ & $(-26.68)$ & $* * *$ & $(-25.14)$ & $* * *$ \\
\hline & & & & odel Statistics & & & & & \\
\hline & observations & 8,515 & & 8,343 & & 8,217 & & 8,187 & \\
\hline & Censored & 127 & & 129 & & 136 & & 138 & \\
\hline & Uncensored & 8,388 & & 8,214 & & 8,081 & & 8,049 & \\
\hline & LR chi2 & 941.41 & & 884.9 & & 927.9 & & 867.7 & \\
\hline & Prob > chi2 & 0.0000 & & 0.0000 & & 0.0000 & & 0.0000 & \\
\hline & Log likelihood & $-130,919$ & & $-130,141$ & & $-129,180$ & & $-129,480$ & \\
\hline & Pseudo R2 & 0.0036 & & 0.0034 & & 0.0036 & & 0.0033 & \\
\hline
\end{tabular}




\begin{tabular}{|c|c|c|c|c|c|c|c|c|c|}
\hline \multicolumn{10}{|c|}{ Tobit regression results: Model 2 Panel C (zero equity in 2008) } \\
\hline & dependent & 2008 & & 2008 & & 2008 & & 2008 & \\
\hline & independent & 2005Q1 & & 2006Q1 & & 2007Q1 & & 2008Q1 & \\
\hline \multirow[t]{2}{*}{1} & cons_devlp & $-11,747$ & & $-14,120$ & & $-17,091$ & & $-18,994$ & \\
\hline & & $(-8.12)$ & $* * *$ & $(-8.48)$ & $* * *$ & $(-9.10)$ & $* * *$ & $(-8.78)$ & $* * *$ \\
\hline \multirow[t]{2}{*}{2} & comm_real & $-10,686$ & & $-12,869$ & & $-15,771$ & & $-17,201$ & \\
\hline & & $(-8.98)$ & $* * *$ & $(-8.49)$ & $* * *$ & $(-8.88)$ & $* * *$ & $(-8.69)$ & $* * *$ \\
\hline \multirow[t]{2}{*}{3} & mul_family & $-12,719$ & & $-17,398$ & & $-7,734$ & & $-1,735$ & \\
\hline & & $(-4.58)$ & $* * *$ & $(-4.77)$ & $* * *$ & $(-1.81)$ & $*$ & $(-0.39)$ & \\
\hline \multirow[t]{2}{*}{4} & sig_family & $-4,041$ & & $-4,445$ & & $-4,924$ & & $-5,697$ & \\
\hline & & $(-3.92)$ & $* * *$ & $(-3.35)$ & $* * *$ & $(-3.15)$ & $* * *$ & $(-3.24)$ & $* * *$ \\
\hline \multirow[t]{2}{*}{5} & ciloan & 7,997 & & 10,297 & & 13,115 & & 13,689 & \\
\hline & & (3.63) & $* * *$ & (3.74) & $* * *$ & $(4.03)$ & $* * *$ & (3.79) & $* * *$ \\
\hline \multirow[t]{2}{*}{6} & capital & 25,642 & & 28,553 & & 33,897 & & 30,910 & \\
\hline & & $(8.70)$ & $* * *$ & $(8.45)$ & $* * *$ & $(9.27)$ & $* * *$ & (7.58) & $* * *$ \\
\hline \multirow[t]{2}{*}{7} & size & 410,657 & & 481,809 & & 563,952 & & 602,690 & \\
\hline & & (30.76) & $* * *$ & (28.83) & $* * *$ & $(29.28)$ & $* * *$ & (28.38) & $* * *$ \\
\hline \multirow[t]{2}{*}{8} & roa & $-14,230$ & & $-4,593$ & & 5,076 & & $-22,072$ & \\
\hline & & $(-1.39)$ & & $(-0.35)$ & & $(0.32)$ & & $(-1.52)$ & \\
\hline \multirow[t]{10}{*}{9} & _cons & $-4,402,370$ & & $-5,181,992$ & & $-6,142,342$ & & $-6,492,635$ & \\
\hline & & $(-27.54)$ & $* * *$ & $(-25.72)$ & $* * *$ & $(-26.30)$ & $* * *$ & $(-24.81)$ & $* * *$ \\
\hline & \multicolumn{9}{|c|}{ Model Statistics } \\
\hline & observations & \multicolumn{2}{|l|}{8,515} & \multicolumn{2}{|l|}{8,343} & \multicolumn{2}{|l|}{8,217} & 8,187 & \\
\hline & Censored & 22 & & 23 & & 25 & & 23 & \\
\hline & Uncensored & 8,493 & & 8,320 & & 8,192 & & 8,164 & \\
\hline & LR chi2 & 955.63 & & 848.14 & & 899.37 & & 849.68 & \\
\hline & Prob $>$ chi 2 & 0.0000 & & 0.0000 & & 0.0000 & & 0.0000 & \\
\hline & Log likelihood & $-132,425$ & & $-131,585$ & & $-130,756$ & & $-131,151$ & \\
\hline & Pseudo R2 & 0.0036 & & 0.0032 & & 0.0034 & & 0.0032 & \\
\hline & & Tobit regressi & $\mathrm{n} \mathrm{resul}$ & odel 2 Panel & (zero & equity in 2007) & & & \\
\hline & dependent & 2007 & & 2007 & & 2007 & & & \\
\hline & independent & 2005Q1 & & 2006Q1 & & 2007Q1 & & & \\
\hline 1 & cons_devlp & $-11,464$ & & $-14,439$ & & $-17,251$ & & & \\
\hline & & $(-7.94)$ & $* * *$ & $(-8.59)$ & $* * *$ & $(-9.15)$ & $* * *$ & & \\
\hline 2 & comm_real & $-10,791$ & & $-13,635$ & & $-16,533$ & & & \\
\hline & & $(-9.09)$ & $* * *$ & $(-8.90)$ & $* * *$ & $(-9.26)$ & $* * *$ & & \\
\hline 3 & mul_family & $-12,794$ & & $-17,692$ & & $-7,536$ & & & \\
\hline & & $(-4.62)$ & $* * *$ & $(-4.79)$ & $* * *$ & $(-1.75)$ & $*$ & & \\
\hline 4 & sig_family & $-4,070$ & & $-4,677$ & & $-5,094$ & & & \\
\hline & & $(-3.95)$ & $* * *$ & $(-3.48)$ & $* * *$ & $(-3.24)$ & $* * *$ & & \\
\hline 5 & ciloan & 7,937 & & 9,972 & & 12,820 & & & \\
\hline & & (3.61) & $* * *$ & (3.59) & $* * *$ & $(3.92)$ & $* * *$ & & \\
\hline 6 & capital & 25,640 & & 29,214 & & 34,315 & & & \\
\hline & & $(8.71)$ & $* * *$ & $(8.55)$ & $* * *$ & $(9.33)$ & $* * *$ & & \\
\hline 7 & size & 412,343 & & 504,488 & & 584,586 & & & \\
\hline & & $(30.95)$ & $* * *$ & (29.89) & $* * *$ & $(30.21)$ & $* * *$ & & \\
\hline 8 & roa & $-14,980$ & & $-5,884$ & & 4,356 & & & \\
\hline & & $(-1.46)$ & & $(-0.45)$ & & $(0.28)$ & & & \\
\hline 9 & _cons & $-4,416,605$ & & $-5,408,261$ & & $-6,349,244$ & & & \\
\hline & & $(-27.68)$ & $* * *$ & $(-26.57)$ & $* * *$ & (27.06) & $* * *$ & & \\
\hline & & & & del Statistics & & & & & \\
\hline & observations & 8,515 & & 8,343 & & 8217 & & & \\
\hline & Censored & 3 & & 3 & & 2 & & & \\
\hline & Uncensored & 8,512 & & 8,340 & & 8,215 & & & \\
\hline & LR chi2 & 966.28 & & 907.83 & & 954.37 & & & \\
\hline & Prob > chi 2 & 0.0000 & & 0.0000 & & 0.0000 & & & \\
\hline & Log likelihood & $-132,694$ & & $-131,976$ & & $-131,150$ & & & \\
\hline & Pseudo R2 & 0.0036 & & 0.0034 & & 0.0036 & & & \\
\hline
\end{tabular}

Note. t-statistics are in the parentheses. $* * *$ and $* * *$ indicate statistical significance at $10 \%, 5 \%, 1 \%$ level, respectively. All variables are described in Table 1. 
Table A3. Tobit regression results: Model 3

\begin{tabular}{|c|c|c|c|c|c|c|c|c|c|}
\hline \multicolumn{10}{|c|}{ Tobit regression results: Model 3 Panel A (zero equity in 2010) } \\
\hline & \multirow{2}{*}{$\begin{array}{l}\text { dependent } \\
\text { independent }\end{array}$} & \multicolumn{2}{|l|}{2010} & \multicolumn{2}{|l|}{2010} & \multicolumn{2}{|l|}{2010} & \multicolumn{2}{|l|}{2010} \\
\hline & & 2005Q1 & & 2006Q1 & & 2007Q1 & & 2008Q1 & \\
\hline \multirow[t]{2}{*}{1} & realloan & $-10,645$ & & $-13,757$ & & $-16,014$ & & $-18,192$ & \\
\hline & & $(-11.49)$ & $* * *$ & $(-11.77)$ & $* * *$ & $(-12.12)$ & $* * *$ & $(-12.19)$ & $* * *$ \\
\hline \multirow[t]{2}{*}{2} & loangrowth & -444.632 & & -5.098 & & -10.834 & & -10.191 & \\
\hline & & $(-1.28)$ & & $(-0.70)$ & & $(-0.64)$ & & $(-0.10)$ & \\
\hline \multirow[t]{2}{*}{3} & capital & 23,336 & & 26,393 & & 24,571 & & 22,062 & \\
\hline & & $(7.36)$ & $* * *$ & (7.38) & $* * *$ & $(6.65)$ & $* * *$ & $(5.44)$ & $* * *$ \\
\hline \multirow[t]{2}{*}{4} & size & 399,883 & & 496,350 & & 555,312 & & 602,100 & \\
\hline & & (31.13) & $* * *$ & $(30.50)$ & $* * *$ & $(30.15)$ & $* * *$ & (29.69) & $* * *$ \\
\hline \multirow[t]{2}{*}{5} & roa & $-25,860$ & & $-4,103$ & & $-14,195$ & & $-13,310$ & \\
\hline & & $(-2.48)$ & $* *$ & $(-0.40)$ & & $(-1.31)$ & & $(-1.52)$ & \\
\hline \multirow[t]{2}{*}{6} & lossallow & $-26,713$ & & $-23,551$ & & $-30,153$ & & $-11,322$ & \\
\hline & & $(-1.82)$ & $*$ & $(-1.31)$ & & $(-1.69)$ & $*$ & $(-0.62)$ & \\
\hline \multirow[t]{2}{*}{7} & pastdue & 44,759 & & 56,956 & & 54,587 & & 8,972 & \\
\hline & & $(3.66)$ & $* * *$ & $(3.62)$ & $* * *$ & (3.38) & $* * *$ & $(0.69)$ & \\
\hline \multirow[t]{2}{*}{8} & chargeoff & 48,515 & & $-1,079$ & & 77,954 & & 45,902 & \\
\hline & & $(0.43)$ & & $(-0.02)$ & & $(0.78)$ & & $(0.38)$ & \\
\hline \multirow[t]{2}{*}{9} & foreclose & 20,745 & & 77,865 & & 39,364 & & 9,180 & \\
\hline & & $(0.39)$ & & $(1.05)$ & & $(0.57)$ & & $(0.18)$ & \\
\hline \multirow[t]{10}{*}{10} & _cons & $-4,393,884$ & & $-5,475,767$ & & $-6,063,080$ & & $-6,470,373$ & \\
\hline & & $(-27.18)$ & $* * *$ & $(-26.58)$ & $* * *$ & $(-26.21)$ & $* * *$ & $(-25.55)$ & $* * *$ \\
\hline & \multicolumn{9}{|c|}{ Model Statistics } \\
\hline & observations & 8,526 & & 8,352 & & 8,233 & & 8,203 & \\
\hline & Censored & 136 & & 140 & & 146 & & 151 & \\
\hline & Uncensored & 8,390 & & 8,212 & & 8,087 & & 8,052 & \\
\hline & LR chi2 & 980.04 & & 920.3 & & 897.39 & & 893.26 & \\
\hline & Prob > chi 2 & 0.0000 & & 0.0000 & & 0.0000 & & 0.0000 & \\
\hline & Log likelihood & $-131,010$ & & $-130,301$ & & $-129,305$ & & $-129,594$ & \\
\hline & Pseudo R2 & 0.0037 & & 0.0035 & & 0.0035 & & 0.0034 & \\
\hline & & Tobit regress & on rest & Model 3 Pane & $\mathrm{B}(\mathrm{ze}$ & quity in 2009) & & & \\
\hline & dependent & 2009 & & 2009 & & 2009 & & 2009 & \\
\hline & independent & 2005Q1 & & 2006Q1 & & 2007Q1 & & 2008Q1 & \\
\hline 1 & realloan & $-10,575$ & & $-13,531$ & & $-15,717$ & & $-17,145$ & \\
\hline & & $(-11.42)$ & $* * *$ & $(-11.58)$ & $* * *$ & $(-11.90)$ & $* * *$ & $(-11.50)$ & $* * *$ \\
\hline 2 & loangrowth & -462.806 & & -4.992 & & -10.923 & & -12.810 & \\
\hline & & $(-1.33)$ & & $(-0.68)$ & & $(-0.65)$ & & $(-0.13)$ & \\
\hline 3 & capital & 23,556 & & 26,309 & & 24,507 & & 22,328 & \\
\hline & & $(7.43)$ & $* * *$ & (7.36) & $* * *$ & $(6.63)$ & $* * *$ & $(5.51)$ & $* * *$ \\
\hline 4 & size & 395,052 & & 490,002 & & 546,704 & & 593,049 & \\
\hline & & (30.73) & $* * *$ & $(30.10)$ & $* * *$ & (29.66) & $* * *$ & $(29.22)$ & $* * *$ \\
\hline 5 & roa & $-26,092$ & & $-3,374$ & & $-14,341$ & & $-10,814$ & \\
\hline & & $(-2.51)$ & $* *$ & $(-0.33)$ & & $(-1.32)$ & & $(-1.23)$ & \\
\hline 6 & lossallow & $-25,674$ & & $-22,348$ & & $-30,058$ & & $-8,656$ & \\
\hline & & $(-1.75)$ & $*$ & $(-1.24)$ & & $(-1.69)$ & $*$ & $(-0.47)$ & \\
\hline 7 & pastdue & 46,600 & & 58,985 & & 53,899 & & -574 & \\
\hline & & $(3.82)$ & $* * *$ & (3.76) & $* * *$ & (3.34) & $* * *$ & $(-0.04)$ & \\
\hline 8 & chargeoff & 49,731 & & 4,381 & & 90,239 & & 35,290 & \\
\hline & & $(0.44)$ & & $(0.06)$ & & $(0.90)$ & & $(0.29)$ & \\
\hline 9 & foreclose & 16,946 & & 78,819 & & 8,485 & & $-37,453$ & \\
\hline & & $(0.32)$ & & (1.07) & & $(0.12)$ & & $(-0.70)$ & \\
\hline 10 & _cons & $-4,344,448$ & & $-5,413,495$ & & $-5,967,113$ & & $-6,386,750$ & \\
\hline & & $(-26.87)$ & $* * *$ & $(-26.28)$ & $* * *$ & $(-25.78)$ & $* * *$ & $(-25.19)$ & $* * *$ \\
\hline
\end{tabular}




\begin{tabular}{|c|c|c|c|c|c|c|c|c|c|}
\hline \multicolumn{10}{|c|}{ Model Statistics } \\
\hline & observations & \multicolumn{2}{|l|}{8,526} & \multicolumn{2}{|l|}{8,352} & \multicolumn{2}{|l|}{8,233} & \multicolumn{2}{|l|}{8,203} \\
\hline & Censored & 125 & & \multicolumn{2}{|l|}{129} & \multicolumn{2}{|l|}{135} & \multicolumn{2}{|l|}{138} \\
\hline & Uncensored & 8,401 & & \multicolumn{2}{|l|}{8,223} & \multicolumn{2}{|l|}{8,098} & \multicolumn{2}{|l|}{8,065} \\
\hline & LR chi2 & 957.54 & & \multicolumn{2}{|l|}{896.6} & \multicolumn{2}{|l|}{871.29} & 869.04 & \\
\hline & Prob > chi 2 & 0.0000 & & 0.0000 & & 0.0000 & & 0.0000 & \\
\hline & Log likelihood & $-131,179$ & & $-130,473$ & & $-129,479$ & & $-129,799$ & \\
\hline & Pseudo R2 & 0.0036 & & 0.0034 & & 0.0034 & & 0.0033 & \\
\hline & & Tobit regress & on res & Model 3 Pan & $\mathrm{C}(\mathrm{ze}$ & quity in 2008) & & & \\
\hline & dependent & 2008 & & 2008 & & 2008 & & 2008 & \\
\hline & independent & 2005Q1 & & 2006Q1 & & 2007Q1 & & 2008Q1 & \\
\hline 1 & realloan & $-10,015$ & & $-12,743$ & & $-14,785$ & & $-16,640$ & \\
\hline & & $(-10.91)$ & $* * *$ & $(-11.16)$ & $* * *$ & $(-11.40)$ & $* * *$ & $(-11.33)$ & $* * *$ \\
\hline 2 & loangrowth & -437.464 & & -5.038 & & -10.710 & & -11.139 & \\
\hline & & $(-1.27)$ & & $(-0.70)$ & & $(-0.64)$ & & $(-0.11)$ & \\
\hline 3 & capital & 23,436 & & 26,171 & & 24,541 & & 22,185 & \\
\hline & & $(7.45)$ & $* * *$ & (7.48) & $* * *$ & $(6.76)$ & $* * *$ & $(5.55)$ & $* * *$ \\
\hline 4 & size & 398,367 & & 477,448 & & 537,800 & & 588,859 & \\
\hline & & $(31.27)$ & $* * *$ & (29.96) & $* * *$ & $(29.69)$ & $* * *$ & (29.44) & $* * *$ \\
\hline 5 & roa & $-26,710$ & & $-3,399$ & & $-14,720$ & & $-11,028$ & \\
\hline & & $(-2.59)$ & $* * *$ & $(-0.34)$ & & $(-1.38)$ & & $(-1.27)$ & \\
\hline 6 & lossallow & $-24,428$ & & $-19,891$ & & $-27,409$ & & $-8,507$ & \\
\hline & & $(-1.68)$ & $*$ & $(-1.13)$ & & $(-1.56)$ & & $(-0.47)$ & \\
\hline 7 & pastdue & 44,603 & & 54,979 & & 54,896 & & 8,974 & \\
\hline & & $(3.68)$ & $* * *$ & $(3.58)$ & $* * *$ & $(3.47)$ & $* * *$ & $(0.70)$ & \\
\hline 8 & chargeoff & 56,559 & & 4,681 & & 79,907 & & $-1,937$ & \\
\hline & & $(0.50)$ & & $(0.07)$ & & $(0.81)$ & & $(-0.02)$ & \\
\hline 9 & foreclose & 29,576 & & 77,002 & & 14,289 & & 15,736 & \\
\hline & & $(0.57)$ & & $(1.07)$ & & $(0.21)$ & & $(0.31)$ & \\
\hline 10 & _cons & $-4,393,449$ & & $-5,282,251$ & & $-5,891,243$ & & $-6,364,687$ & \\
\hline & & $(-27.41)$ & $* * *$ & $(-26.19)$ & $* * *$ & $(-25.89)$ & $* * *$ & $(-25.47)$ & $* * *$ \\
\hline & & & & Iodel Statisti & & & & & \\
\hline & observations & 8,526 & & 8,352 & & 8,233 & & 8,203 & \\
\hline & Censored & 21 & & 23 & & 25 & & 23 & \\
\hline & Uncensored & 8,505 & & 8,329 & & 8,208 & & 8,180 & \\
\hline & LR chi2 & 983.52 & & 887.65 & & 868.69 & & 868.08 & \\
\hline & Prob > chi 2 & 0.0000 & & 0.0000 & & 0.0000 & & 0.0000 & \\
\hline & Log likelihood & $-132,666$ & & $-131,912$ & & $-131,027$ & & $-131,463$ & \\
\hline & Pseudo R2 & 0.0037 & & 0.0034 & & 0.0033 & & 0.0033 & \\
\hline & & Tobit regress & on rest & Model 3 Pan & $\mathrm{D}(\mathrm{z}$ & quity in 2007) & & & \\
\hline & dependent & 2007 & & 2007 & & 2007 & & & \\
\hline & independent & 2005Q1 & & 2006Q1 & & 2007Q1 & & & \\
\hline 1 & realloan & $-9,893$ & & $-12,620$ & & $-14,639$ & & & \\
\hline & & $(-10.80)$ & $* * *$ & $(-10.93)$ & $* * *$ & $(-11.22)$ & $* * *$ & & \\
\hline 2 & loangrowth & -432.270 & & -5.052 & & -10.695 & & & \\
\hline & & $(-1.26)$ & & $(-0.70)$ & & $(-0.64)$ & & & \\
\hline 3 & capital & 23,582 & & 26,927 & & 25,175 & & & \\
\hline & & $(7.51)$ & $* * *$ & $(7.62)$ & $* * *$ & $(6.90)$ & $* * *$ & & \\
\hline 4 & size & 400,033 & & 497,276 & & 556,416 & & & \\
\hline & & (31.45) & $* * *$ & $(30.88)$ & $* * *$ & $(30.55)$ & $* * *$ & & \\
\hline 5 & roa & $-27,361$ & & $-4,132$ & & $-14,621$ & & & \\
\hline & & $(-2.65)$ & $* * *$ & $(-0.41)$ & & $(-1.36)$ & & & \\
\hline 6 & lossallow & $-24,364$ & & $-20,748$ & & $-28,373$ & & & \\
\hline & & $(-1.67)$ & $*$ & $(-1.16)$ & & $(-1.61)$ & & & \\
\hline 7 & pastdue & 45,030 & & 59,476 & & 58,675 & & & \\
\hline & & $(3.73)$ & $* * *$ & $(3.84)$ & $* * *$ & $(3.68)$ & $* * *$ & & \\
\hline 8 & chargeoff & 56,384 & & 2,255 & & 81,687 & & & \\
\hline & & $(0.50)$ & & $(0.03)$ & & $(0.82)$ & & & \\
\hline 9 & foreclose & 28,279 & & 82,682 & & 30,822 & & & \\
\hline & & $(0.54)$ & & (1.14) & & $(0.45)$ & & & \\
\hline 10 & _cons & $-4,417,416$ & & $-5,528,607$ & & $-6,125,452$ & & & \\
\hline & & $(-27.61)$ & $* * *$ & $(-27.13)$ & $* * *$ & $(-26.78)$ & $* * *$ & & \\
\hline
\end{tabular}




\begin{tabular}{lrrr}
\hline & \multicolumn{3}{c}{ Model Statistics } \\
\hline observations & 8,526 & 8,352 & 8,233 \\
Censored & 3 & 3 & 2 \\
Uncensored & 8,523 & 8,349 & 8,231 \\
LR chi2 & 993.01 & 934.68 & 912.92 \\
Prob > chi2 & 0.0000 & 0.0000 & 0.0000 \\
Log likelihood & $-132,921$ & $-132,303$ & $-131,426$ \\
Pseudo R2 & 0.0037 & 0.0035 & 0.0035 \\
\hline
\end{tabular}

Note. t-statistics are in the parentheses. $*, * *$ and $* * *$ indicate statistical significance at $10 \%, 5 \%, 1 \%$ level, respectively. All variables are described in Table 1.

Table A4. Tobit regression results: Model 5

\begin{tabular}{|c|c|c|c|c|c|c|c|c|c|}
\hline \multicolumn{10}{|c|}{ Tobit regression results: Model 5 Panel A (zero equity in 2010) } \\
\hline & \multirow{2}{*}{$\begin{array}{l}\text { dependent } \\
\text { independent }\end{array}$} & \multicolumn{2}{|l|}{2010} & \multicolumn{2}{|l|}{2010} & \multicolumn{2}{|l|}{2010} & \multicolumn{2}{|l|}{2010} \\
\hline & & 2005Q1 & & 2006Q1 & & 2007Q1 & & 2008Q1 & \\
\hline \multirow[t]{2}{*}{1} & realloan & -12858.08 & & -15097.09 & & -17703.75 & & -18777.59 & \\
\hline & & $(-9.88)$ & $* * *$ & $(-9.07)$ & $* * *$ & $(-9.56)$ & $* * *$ & $(-8.40)$ & $* * *$ \\
\hline \multirow[t]{2}{*}{2} & capital & 10,255 & & 13,289 & & 12,482 & & 8,840 & \\
\hline & & $(3.54)$ & $* * *$ & $(4.02)$ & $* * *$ & $(3.51)$ & $* * *$ & $(2.23)$ & $* *$ \\
\hline \multirow[t]{2}{*}{3} & size & 418,226 & & 512,689 & & 572,429 & & 631,747 & \\
\hline & & (32.04) & $* * *$ & (30.69) & $* * *$ & (30.18) & $* * *$ & (30.46) & $* * *$ \\
\hline \multirow[t]{2}{*}{4} & roa & $-11,192$ & & 6,722 & & $-4,078$ & & $-8,813$ & \\
\hline & & $(-1.16)$ & & $(0.75)$ & & $(-0.43)$ & & $(-1.05)$ & \\
\hline \multirow[t]{2}{*}{5} & loan_ast & $-9,980$ & & $-12,780$ & & $-9,993$ & & $-19,538$ & \\
\hline & & $(-4.35)$ & $* * *$ & $(-4.48)$ & $* * *$ & $(-3.27)$ & $* * *$ & $(-5.55)$ & $* * *$ \\
\hline \multirow[t]{2}{*}{6} & lossallow & $-17,836$ & & $-26,159$ & & $-14,076$ & & $-7,054$ & \\
\hline & & $(-1.72)$ & $*$ & $(-1.46)$ & & $(-0.86)$ & & $(-0.39)$ & \\
\hline \multirow[t]{2}{*}{7} & pastdue & 38,239 & & 51,005 & & 48,948 & & 4,107 & \\
\hline & & $(3.22)$ & $* * *$ & (3.24) & $* * *$ & (3.09) & $* * *$ & $(0.35)$ & \\
\hline \multirow[t]{2}{*}{8} & MBS & $-4,869$ & & $-5,424$ & & $-7,340$ & & $-7,923$ & \\
\hline & & $(-2.40)$ & $* *$ & $(-1.88)$ & $*$ & $(-2.12)$ & $* *$ & $(-2.21)$ & $* *$ \\
\hline \multirow[t]{2}{*}{9} & debt_sec & $-14,700$ & & $-17,848$ & & $-16,298$ & & $-26,247$ & \\
\hline & & $(-6.82)$ & $* * *$ & $(-6.62)$ & $* * *$ & $(-5.44)$ & $* * *$ & $(-7.35)$ & $* * *$ \\
\hline \multirow[t]{2}{*}{10} & pi_grow & $-572,115$ & & $-3,072,812$ & & 491,848 & & 606,637 & \\
\hline & & $(-0.48)$ & & $(-1.50)$ & & $(0.15)$ & & $(0.27)$ & \\
\hline \multirow[t]{2}{*}{11} & hpindex_sa & $-1,145$ & & $-1,392$ & & $-2,190$ & & $-2,164$ & \\
\hline & & $(-2.08)$ & $* *$ & $(-2.32)$ & $* *$ & $(-3.29)$ & $* * *$ & $(-2.50)$ & $* *$ \\
\hline \multirow[t]{2}{*}{12} & _cons & $-3,145,858$ & & $-3,832,873$ & & $-4,566,586$ & & $-4,334,350$ & \\
\hline & & $(-12.43)$ & $* * *$ & $(-12.25)$ & $* * *$ & $(-13.22)$ & $* * *$ & $(-10.73)$ & $* * *$ \\
\hline \multicolumn{10}{|c|}{ Model Statistics } \\
\hline \multicolumn{2}{|r|}{ observations } & \multicolumn{2}{|l|}{8,563} & 8,400 & & 8,274 & & 8,238 & \\
\hline & Censored & 137 & & 144 & & 147 & & 151 & \\
\hline & Uncensored & 8,426 & & 8,256 & & 8,127 & & 8,087 & \\
\hline & LR chi2 & 1,061 & & 983 & & 947 & & 988 & \\
\hline & Prob > chi 2 & 0.0000 & & 0.0000 & & 0.0000 & & 0.0000 & \\
\hline & Log likelihood & $-131,529$ & & $-130,951$ & & $-129,902$ & & $-130,094$ & \\
\hline & Pseudo R2 & 0.0040 & & 0.0037 & & 0.0036 & & 0.0038 & \\
\hline & & bit regression & esults: & del 5 Panel 1 & (zero & ity in 2009) & & & \\
\hline & dependent & 2009 & & 2009 & & 2009 & & 2009 & \\
\hline & independent & 2005Q1 & & 2006Q1 & & 2007Q1 & & 2008Q1 & \\
\hline 1 & realloan & -13295.26 & & -15355.35 & & -17873.44 & & -18785.66 & \\
\hline & & $(-10.18)$ & $* * *$ & $(-9.23)$ & $* * *$ & $(-9.66)$ & $* * *$ & $(-8.41)$ & $* * *$ \\
\hline 2 & capital & 9,950 & & 13,590 & & 12,377 & & 9,089 & \\
\hline & & (3.43) & $* * *$ & $(4.12)$ & $* * *$ & (3.48) & $* * *$ & $(2.29)$ & $* *$ \\
\hline 3 & size & 413,480 & & 505,488 & & 563,298 & & 622,031 & \\
\hline & & $(31.67)$ & $* * *$ & $(30.26)$ & $* * *$ & (29.69) & $* * *$ & (29.97) & $* * *$ \\
\hline 4 & roa & $-9,740$ & & 5,409 & & $-3,962$ & & $-6,000$ & \\
\hline & & $(-1.01)$ & & $(0.61)$ & & $(-0.41)$ & & $(-0.71)$ & \\
\hline
\end{tabular}




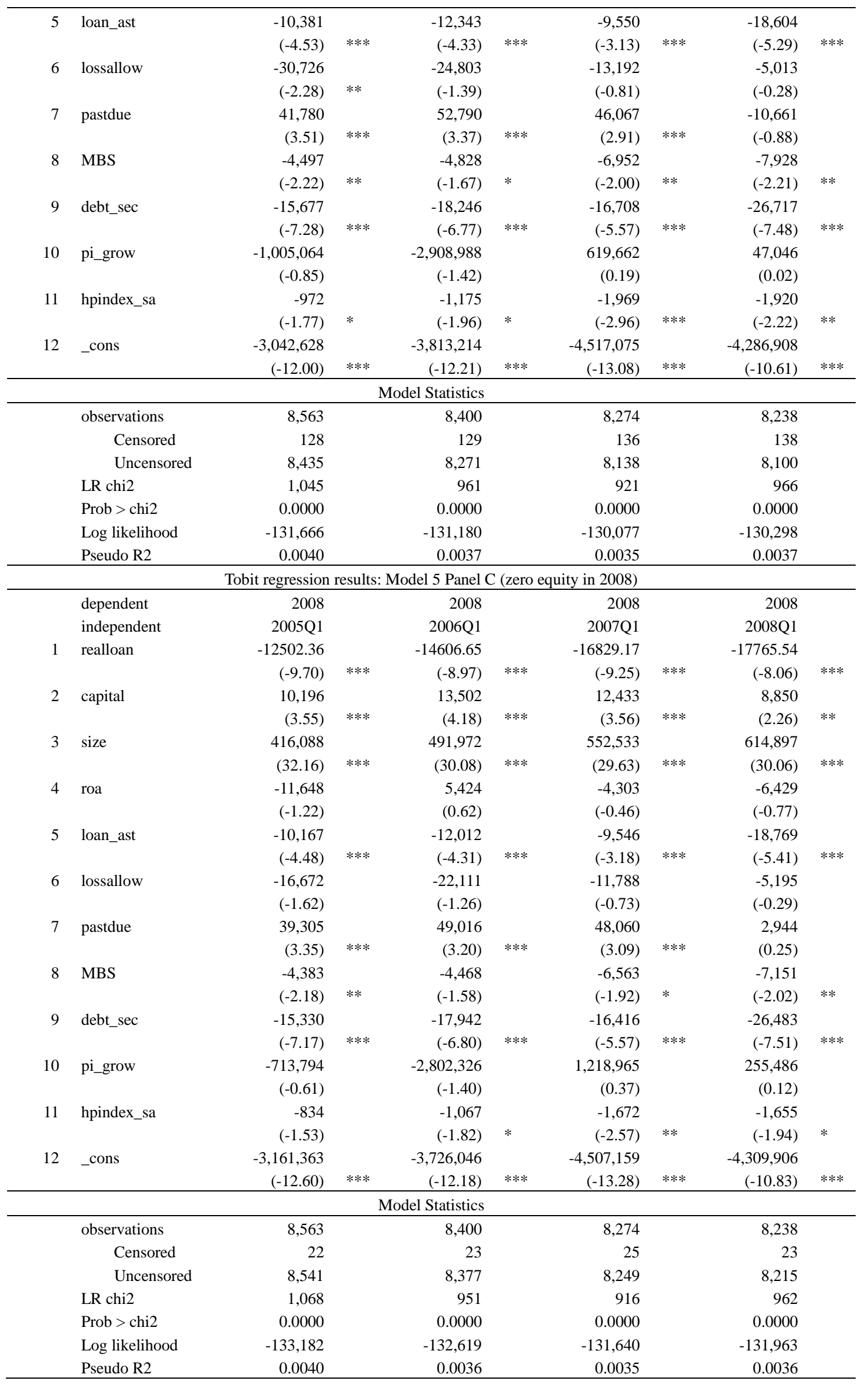




\begin{tabular}{|c|c|c|c|c|c|c|c|}
\hline \multicolumn{8}{|c|}{ Tobit regression results: Model 5 Panel D (zero equity in 2007) } \\
\hline & dependent & 2007 & & 2007 & & 2007 & \\
\hline & independent & 2005Q1 & & 2006Q1 & & 2007Q1 & \\
\hline \multirow[t]{2}{*}{1} & realloan & -12380.29 & & -14353.91 & & -16572.16 & \\
\hline & & $(-9.62)$ & $* * *$ & $(-8.72)$ & $* * *$ & $(-9.06)$ & $* * *$ \\
\hline \multirow[t]{2}{*}{2} & capital & 10,276 & & 13,821 & & 12,689 & \\
\hline & & $(3.59)$ & $* * *$ & $(4.23)$ & $* * *$ & $(3.61)$ & $* * *$ \\
\hline \multirow[t]{2}{*}{3} & size & 417,588 & & 512,197 & & 571,823 & \\
\hline & & $(32.33)$ & $* * *$ & $(31.00)$ & $* * *$ & $(30.50)$ & $* * *$ \\
\hline \multirow[t]{2}{*}{4} & roa & $-12,273$ & & 4,997 & & $-4,351$ & \\
\hline & & $(-1.29)$ & & $(0.57)$ & & $(-0.46)$ & \\
\hline \multirow[t]{2}{*}{5} & loan_ast & $-10,187$ & & $-12,703$ & & $-10,117$ & \\
\hline & & $(-4.49)$ & $* * *$ & $(-4.50)$ & $* * *$ & $(-3.35)$ & $* * *$ \\
\hline \multirow[t]{2}{*}{6} & lossallow & $-16,715$ & & $-23,609$ & & $-12,486$ & \\
\hline & & $(-1.63)$ & & $(-1.33)$ & & $(-0.77)$ & \\
\hline \multirow[t]{2}{*}{7} & pastdue & 39,552 & & 54,426 & & 53,089 & \\
\hline & & (3.38) & $* * *$ & $(3.52)$ & $* * *$ & (3.39) & $* * *$ \\
\hline \multirow[t]{2}{*}{8} & MBS & $-4,413$ & & $-4,999$ & & $-7,131$ & \\
\hline & & $(-2.20)$ & $* *$ & $(-1.75)$ & $*$ & $(-2.08)$ & $* *$ \\
\hline \multirow[t]{2}{*}{9} & debt_sec & $-15,384$ & & $-18,283$ & & $-16,714$ & \\
\hline & & $(-7.21)$ & $* * *$ & $(-6.85)$ & $* * *$ & $(-5.64)$ & $* * *$ \\
\hline \multirow[t]{2}{*}{10} & pi_grow & $-598,309$ & & $-2,992,164$ & & 916,973 & \\
\hline & & $(-0.51)$ & & $(-1.48)$ & & $(0.28)$ & \\
\hline \multirow[t]{2}{*}{11} & hpindex_sa & -794 & & -902 & & $-1,584$ & \\
\hline & & $(-1.46)$ & & $(-1.52)$ & & $(-2.42)$ & $* *$ \\
\hline \multirow[t]{2}{*}{12} & _cons & $-3,187,616$ & & $-3,953,276$ & & $-4,717,640$ & \\
\hline & & $(-12.73)$ & $* * *$ & $(-12.79)$ & $* * *$ & $(-13.83)$ & $* * *$ \\
\hline \multicolumn{8}{|c|}{ Model Statistics } \\
\hline & observations & 8,563 & & 8,400 & & 8,274 & \\
\hline & Censored & 3 & & 3 & & 2 & \\
\hline & Uncensored & 8,560 & & 8,397 & & 8,272 & \\
\hline & LR chi2 & 1,078 & & 999 & & 961 & \\
\hline & Prob > chi 2 & 0.0000 & & 0.0000 & & 0.0000 & \\
\hline & Log likelihood & $-133,452$ & & $-133,010$ & & $-132,039$ & \\
\hline & Pseudo R2 & 0.0040 & & 0.0037 & & 0.0036 & \\
\hline
\end{tabular}

Note. t-statistics are in the parentheses. *,** and *** indicate statistical significance at $10 \%, 5 \%, 1 \%$ level, respectively. All variables are described in Table 1.

\section{Copyrights}

Copyright for this article is retained by the author(s), with first publication rights granted to the journal.

This is an open-access article distributed under the terms and conditions of the Creative Commons Attribution license (http://creativecommons.org/licenses/by/4.0/). 